\title{
Topic-Based Influence Computation in Social Networks Under Resource Constraints
}

\author{
Kaan Bingöl ${ }^{\circledR}$, Bahaeddin Eravcı ${ }^{\circledR}$, Çağrı Özgenç Etemoğlu, Hakan Ferhatosmanoğlu, and Buğra Gedik ${ }^{\circledR}$
}

\begin{abstract}
As social networks are constantly changing and evolving, methods to analyze dynamic social networks are becoming more important in understanding social trends. However, due to the restrictions imposed by the social network service providers, the resources available to fetch the entire contents of a social network are typically very limited. As a result, analysis of dynamic social network data requires maintaining an approximate copy of the social network for each time period, locally. In this paper, we study the problem of dynamic network and text fetching with limited probing capacities, for identifying and maintaining influential users as the social network evolves. We propose an algorithm to probe the relationships (required for global influence computation) as well as posts (required for topic-based influence computation) of a limited number of users during each probing period, based on the influence trends and activities of the users. We infer the current network based on the newly probed user data and the last known version of the network maintained locally. Additionally, we propose to use link prediction methods to further increase the accuracy of our network inference. We employ PageRank as the metric for influence computation. We illustrate how the proposed solution maintains accurate PageRank scores for computing global influence, and topic-sensitive weighted PageRank scores for topic-based influence. The latter relies on a topic-based network constructed via weights determined by semantic analysis of posts and their sharing statistics. We evaluate the effectiveness of our algorithms by comparing them with the true influence scores of the full and up-to-date version of the network, using data from the micro-blogging service Twitter. Results show that our techniques significantly outperform baseline methods ( 80 percent higher accuracy for network fetching and 77 percent for text fetching) and are superior to state-of-the-art techniques from the literature (21 percent higher accuracy).
\end{abstract}

Index Terms-Estimation, evolving social networks, dynamic network probing, incomplete graphs, topic-sensitive influence

\section{INTRODUCTION}

A NALYSIS of social networks have attracted significant research attention in recent years due to the popularity of online social networks among users and the vast amount of social network data publicly available for analysis. Applications of social network analyses are abound, such as influential user detection, community detection, information diffusion, network modeling, user recommendation, to name a few.

Influential user detection is a key social analysis used for opinion mining, targeted advertising, churn prediction, and word-of-mouth marketing. Social networks are dynamic and constantly evolving via user interactions. Accordingly, the influence of users within the network are also dynamic. Beyond the current influence of users, tracking the influence trends provides greater insights for deeper analysis. By combining the patterns of the past with the current information, comprehensive analysis on customers, marketing plans, and business models can be performed more accurately. For example, forecasting future user influences can be used to detect 'rising stars', who can be employed in upcoming on-line advertisement campaigns.

- K. Bingol, B. Eravcl, H. Ferhatosmanoğlu, and B. Gedik are with the Department of Computer Engineering, Bilkent University, Bilkent, Ankara 6800, Turkey. E-mail: kbingol@icloud.com, beravci@gmail.com, \{hakan, bgedik\}@cs.bilkent.edu.tr.

- Ç.Ö. Etemoğlu is with Türk Telekom, Istanbul 07170, Turkey. E-mail:CagriOzgenc.Etemoglu@turktelekom.com.tr.

Manuscript received 3 Dec. 2015; revised 14 Apr. 2016; accepted 8 Oct. 2016. Date of publication 21 Oct. 2016; date of current version 11 Dec. 2019.

Digital Object Identifier no. 10.1109/TSC.2016.2619688
In this paper, we address the problem of identifying and tracking influential users in dynamic social networks under real-world data acquisition resource limits. The current approaches for influence analysis mostly assume that the graph structure is static, or even when it is dynamic, the data is completely known and stored in a local database. However, in many cases, analysts are third-party clients and do not own the data. They cannot keep the data completely fresh as changes happen, since it is typically gathered from a service provider with limitations on resources or even on the amount of data provided. Third-party data acquisition tools access the data via rate-limited APIs, which constraint the fetching capacity of clients. These externally enforced limits prevent the collection of entire up-to-date data within a predetermined period. To this end, we present an effective solution to rate-limited fetching of evolving network relations and user posts. Our system maintains a local, partially fresh copy of the data and calculates influence scores based on inferred network and text data. The proposed solution probes limited number of active users whose influence scores are changing significantly within the network. By combining previous and the newly probed network data, we are able to calculate the current user influences accurately. The local network copy is maintained while consuming resources within allowed limits, and at the same time, influence values of the users are computed as accurately as possible.

While computing and maintaining influence scores, we consider both global and topic-based influence. Active and influential users mostly affect the general opinion with respect to their topics of authority. For instance, a company marketing sports goods will be interested in locating users 
who have high influence in sports, rather than the global community. While this leads us to consider topic-based analyses in our problem setting, general influence scores of users are still of interest as well. For instance, a politician would prefer a broader audience and identify a list of globally influential users to promote her cause. In our system, we utilize both global and topic-based networks and compute global as well as topic-based influences.

To demonstrate the effectiveness of our solutions, we use Twitter [1]. Twitter is a good fit for research on dynamic user influence detection due to its large user base and highly dynamic user activity. One can collect two-way friendship relations as well as one-way follow, re-tweet (RT), and favorite (FAV) relations via the publicly available Twitter APIs. These APIs have well-defined resource limits [2], which motivates the need for our probing algorithms. We calculate PageRank [3] on the Twitter network as the influence score for the users. To generate topic-based influence scores, we adapt the weighted PageRank [4], and adjust the initial scores and transition probabilities based on topic relevance scores of the users. The topic relevance scores are computed based on user posts, using text mining techniques, as well as the re-tweet and favorite counts of the tweets.

To further improve the accuracy of our network inference, we perform link prediction using trends on user relationships. The proposed solution shows increased accuracy on Twitter data when compared with other methods from the literature. Estimated network structure is shown to be very close to the actual up-to-date network, with respect to influential users. The proposed solutions address not only the limitations of data fetching via public APIs, but also local processing when the resources are limited to fetch the entire data. We summarize our major contributions as follows:

- We estimate global and topic-based influence of users within a dynamic social network. For topic-based influence estimation, we construct topic-based networks via semantic analyses of tweets and the use of re-tweet and favorite statistics for the topic of interest.

- We propose efficient algorithms for collecting dynamic network and text data, under limited resource availability. We leverage both latest known user influence values, as well as the past user influence trends in our probing strategy. We further improve our probing techniques by applying link prediction methods.

- We evaluate our proposed algorithms and compare results to several alternatives from the literature. The experimental results for relationship fetching used for influence estimation show that the proposed algorithms perform 80 percent better than the baseline methods, and 21 percent better than the state-ofthe-art method from the literature in terms of mean squared error. For tweet fetching methods used for topic-based influence detection, our algorithms perform 77 percent better than the alternative baselines in terms of the Jaccard similarity measure.

The rest of this paper is organized as follows. Section 2 describes the resource constraint problem for data collection. Section 3 gives the overall system architecture and presents influence estimation techniques. Section 4 explains algorithms and strategies proposed for the network and text fetching problems. Section 5 discusses results obtained from experiments run on real data. Section 7 discusses related work. Section 8 concludes the paper.

\section{Problem Definition}

Our goal is to determine top-m influential users in the network, under a constrained probing setting. Among various methods to calculate a user's influence in the network, we have chosen PageRank based methods, since PageRank is well understood and used widely in the literature for various network structures. While computing influence, PageRank naturally considers the number of followers a user has, but more importantly it takes into account the topological place of the user within the network. Therefore, we assume that a user's influence in the network corresponds to its PageRank score. As a result, the top-m influential user determination problem turns into identifying the top-m users with the highest PageRank scores. One can also utilize other approaches that can outperform PageRank for estimating social influence within our framework. These approaches need to produce a single score that will be calculated periodically for every user.

PageRank score calculation requires having access to all the relationships present between the users of the network. This means that we need to have the complete network data to compute exact PageRank scores. Moreover, if the network is dynamic, the calculation needs up-to-date network data for each time step in order to perform accurate influence analysis.

Our system continuously collects social network data (relations, tweets, re-tweets, etc.) via the publicly available Twitter API. Twitter enforces certain limitations on data acquisition using the Twitter APIs. There are different limitations for different types of data acquisition requests:

- Relations ${ }^{1}: 15$ calls per 15 minutes, where each call is for retrieving a user's relations. Moreover, if the user has more than $5 \mathrm{~K}$ followers, we need an extra call for each additional $5 \mathrm{~K}$ followers. This means that we can update relations with a maximum rate of 1 user per minute $\left(R_{\text {rel }}=1 \mathrm{user} / \mathrm{min}\right)$.

- Tweets: 180 calls per 15 minutes, where each call is for retrieving a user's tweets. Moreover, if the user has more than 200 tweets, we need an extra call for each additional 200 tweets. This means that we can update tweets with a maximum rate of 12 users per minute $\left(R_{t w t}=12 \mathrm{user} / \mathrm{min}\right)^{2}$

Assuming that we update the network with a period of $P$ days, we need the following condition to hold, in order to be able to capture the entire network of relations

$$
\text { Number of Users } \leq R_{\text {rel }} \cdot P \cdot 1440 .
$$

1. For the relations, Twitter provides two different APIs: one for fetching the user IDs for every user following a specified user, and another for fetching the user IDs for every user a specified user is following. Our system utilizes both APIs, however for brevity of the rate limit calculations details are omitted.

2. The best case, if all users have $\leq 200$ tweets on their timelines. 
For getting the recent tweets of the users, we need

$$
\text { Number of Users } \leq R_{t w t} \cdot P \cdot 1440 .
$$

One can easily calculate that for a network as small as $250 \mathrm{~K}$ users, we need 174 days to update the complete network in the best case. ${ }^{3}$ This analysis shows that the rate limits hinder the timeliness of the data collection process, which in turn affects the timeliness of the calculation process to find and track influential users in the network. Furthermore, Twitter is a highly dynamic network that evolves at a fast rate, which means that refreshing the network infrequently will result in significant degradation in the accuracy of the influence scores. Current resource limits prohibit the system to collect the network data in a reasonable period of time. Therefore, the evolving network's relationships and the tweet sets are not fully observable at every analysis time step.

To overcome this limitation, we propose to determine a small subset of users during each data collection period, whose information is to be updated. This data collection process, which does not violate the rate limits of the API, is sufficient to maintain an approximate network with a reasonable data collection period, while at the same time providing good accuracy for the estimated influence scores.

We apply the concept of probing for efficient fetching of the dynamic network and the user tweets. We denote a network at time $t$ as $G_{t}=\left\{V_{t}, E_{t}\right\}$, where $V_{t}$ is the set of users and $E_{t} \subset V_{t} \times V_{t}$ is the set of edges representing the follower relationship within the network. In other words, $(u, v) \in E_{t}$ means that the user $u \in V_{t}$ is following the user $v \in V_{t}$. Our model uses an evolving set of networks in time, represented as $\left\{G_{t} \mid 0 \leq t \leq T\right\}$. However, we assume that we have fully ${ }^{4}$ observed the network only at time $t=0$. $G_{t}$ where $t>0$, can only be observed partially by probing. At each time period, we use an algorithm to determine a subset of $k$ users and probe them via API calls. We then update the existing local network with the new information obtained from the probed users. In effect, we maintain a partially observed network $G_{t}^{\prime}$, which can potentially differ from the actual network $G_{t}$. Larger $k$ values bring the partial network $G_{t}^{\prime}$ closer to the actual network $G_{t}$. However, using large $k$ values is not feasible due to rate limits outlined earlier. Our probing strategy should select a relatively small number of users to probe, so that the data collection process can be completed within the period $P$ (as determined by Eq. (1)). Furthermore, these probed users should bring the most value in terms of performing accurate influence detection.

Dynamic Network Fetching Problem Definition: We assume that complete network information is available only at time 0 , i.e., $G_{0}$ is known. The problem is defined as determining a subset of users of size $k$ at time $t$ (where $t \geq 1$ ), denoted by $U_{t}^{N} \subset V_{t}$ s.t. $\left|U_{t}^{N}\right|=k$, by analyzing the local graph $G_{t-1}^{\prime}$. The system will retrieve the partial graph related with $U_{t}^{N}$, which is denoted as $G_{t}^{p}\left(U_{t}^{N}\right)=\left(V_{t}^{p}, E_{t}^{p}\right)$ where $V_{t}^{p}=U_{t}^{N}$, and update the relationships of the users included in this

3. If all users have $\leq 5 \mathrm{~K}$ followers, requiring a single call per user.

4. The initial probing of the network can be accelerated via the use of multiple cooperating fetchers. However, this is clearly not a sustainable and feasible approach for continued probing of the network, as it requires large number of accounts, which are subject to bot detection and suspension.

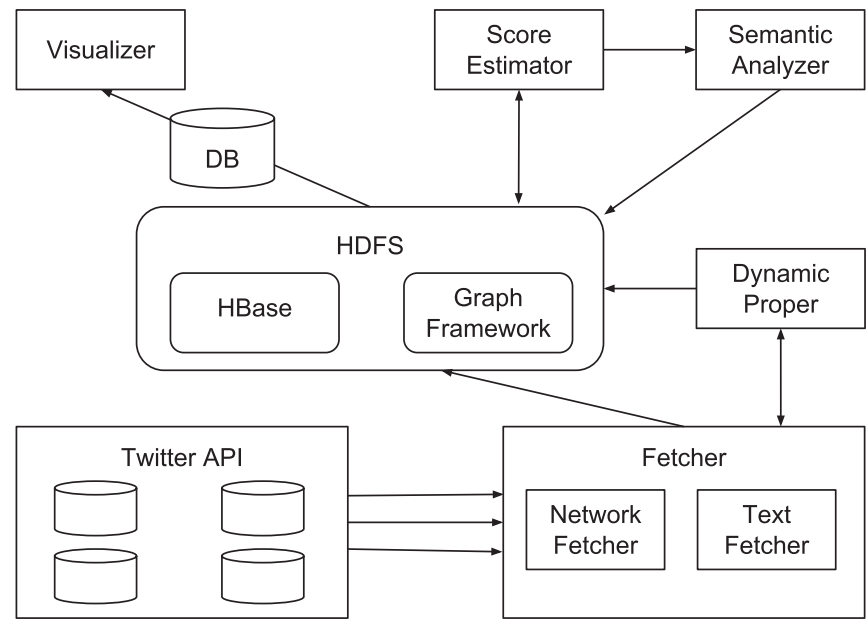

Fig. 1. Overall system architecture.

subset to construct the local network at time $t$, that is $G_{t}^{\prime}$. We define the additions and deletions to the network as $\Sigma\left(U_{t}^{N}\right)=G_{t-1}^{\prime} \backslash G_{t}^{p}\left(U_{t}^{N}\right)$ and $\Delta\left(U_{t}^{N}\right)=G_{t}^{p}\left(U_{t}^{N}\right) \backslash G_{t-1}^{\prime}$, respectively. Using these definitions we can find the network at time $t$, as $G_{t}^{\prime}=G_{t-1}^{\prime} \cup \Sigma\left(U_{t}^{N}\right) \backslash \Delta\left(U_{t}^{N}\right)$.

We aim to choose $U_{t}^{N}$ such that the influence scores of the estimated network $G_{t}^{\prime}$ will be as close as possible to the true scores of the real network $G_{t}$. We summarize the problem as follows:

$$
\begin{array}{r}
\operatorname{argmin}_{U_{t}^{N}}\left(\text { Influence }\left(G_{t}^{\prime}\right)-\text { Influence }\left(G_{t}\right)\right) \\
\text { where } G_{t}^{\prime}=G_{t-1}^{\prime} \cup \Sigma\left(U_{t}^{N}\right) \backslash \Delta\left(U_{t}^{N}\right) .
\end{array}
$$

The final objective is to estimate the PageRank scores $P R_{v}^{\prime}(t), \forall v \in G_{t}$ as accurately as possible, using partial knowledge about $G_{t-1}$, that is $G_{t-1}^{\prime}$, since we have used Pagerank as the indication of influence in this study.

Dynamic Tweet Fetching Problem Definition: Given the tweets $T_{0}$ of all users in the network at time 0 , the problem is defined as determining a subset of users of size $k$ at time $t$ (where $t \geq 1$ ), denoted by $U_{t}^{T} \subset V_{t}$ s.t. $\left|U_{t}^{T}\right|=k$, by analyzing the tweet set $T_{t-1}^{\prime}$ and the local graph $G_{t-1}^{\prime}$. The system will retrieve the partial tweet set for $U_{t}^{T}$, which is denoted as $T_{t}^{p}\left(U_{t}^{T}\right)=\left(V_{t}^{p}, E_{t}^{p}\right)$ where $V_{t}^{p}=U_{t}^{T}$, and update the tweet sets of the users included in this subset to construct the tweet set at time $t$, that is $T_{t}^{\prime}$.

In this paper, we mainly focused on effective ways of handling edge additions and removals. However, node changes are also dynamically happening in the social network. The system handles node changes by periodically renewing the seed list. ${ }^{5}$ For brevity and in order to focus on the more prominent issue of edge additions and removals, seed list updates are not performed as part of our experiments.

\section{Overall System Architecture}

In this section we briefly describe our system architecture, which depicted in Fig. 1.

5. This period is a configuration that can be adjusted by a system administrator. 


\subsection{Social Network Data Collection}

We use the Twitter network and tweets to analyze user influence. A Twitter network is a directed, unweighted graph where the nodes represent users and the edges denote follower relationships in Twitter. When a user $u$ follows another user $v, u$ can see what $v$ is posting, and thus $v$ is considered to have an influence on $u$. Moreover, the user $u$ also would have an effect on $v^{\prime}$ s influence, since the number of people $v$ reaches would potentially increase. This interaction has an effect on both users' influence scores. In order to construct our network, we first determine a small set of users called the core seeds. For illustration, we started with some popular Turkish Twitter accounts including newspapers, TV channels, politicians, sport teams, and celebrities. Second, we collect one- hop relations of the core seeds and add the unique users to a set called the main seeds. We iterate once more to collect one-hop relations of the main seeds with a filter to avoid unrelated and inactive users. This filter has three conditions: $a$ ) a user must have at least five followers, $b$ ) a user must have at least one tweet within the last three months, and $c$ ) the tweet language of a user must be Turkish. As a result of this process, we have determined our seed users set, which includes approximately 2.8 million unique users. In the final step of the data collection phase, we acquire the relations of the seed users to determine $G_{0}$, that is the social network graph at time 0 . Furthermore, we collect tweets of the seed users in order to construct $T_{0}$, that the tweet set at time 0 .

We implemented the proposed methods using a distributed system with HBase and HDFS serving as the database and file system backends. The system consists of six main parts: a) local copy of the social network data on HDFS, $b$ ) data fetcher, c) dynamic prober, d) score estimator, e) semantic analyzer, and f) visualizer. Data fetcher component, as the name implies, fetches the data (network relations and tweets) via rate-limited Twitter APIs, periodically. Dynamic prober makes a dynamic probing analysis, decides which users are going to be fetched and notifies data fetcher to bring the information, accordingly. Score estimator calculates users' influence and the related parameters of the proposed algorithms, which are essential parts of the probing method. Semantic analyzer performs keyword extraction and calculates the related parameters for constructing topicbased networks. Finally, visualizer provides a graphical user interface for result analysis.

\subsection{Score Analysis}

We calculate influence scores of users based on their relationships and the overall impact of their tweets in the network. We analyze topic activities of the users from their tweets and determine topic-based user influence scores. Overall, we are using two types of scores, namely global influence and topic-based influence, which can be interpreted together for a more detailed analyses.

Global Influence Score. This score is a measure of the user's overall influence within the network. For this purpose we use the PageRank $(P R)$ algorithm. PageRank value $P R_{v}(t)$ at time $t$ for a user $v \in G_{t}$ directly corresponds to the global influence score of it and will be used interchangeably throughout the paper.

Fig. 2 illustrates the evolving nature of the influence score by showing the global and topic-based influence

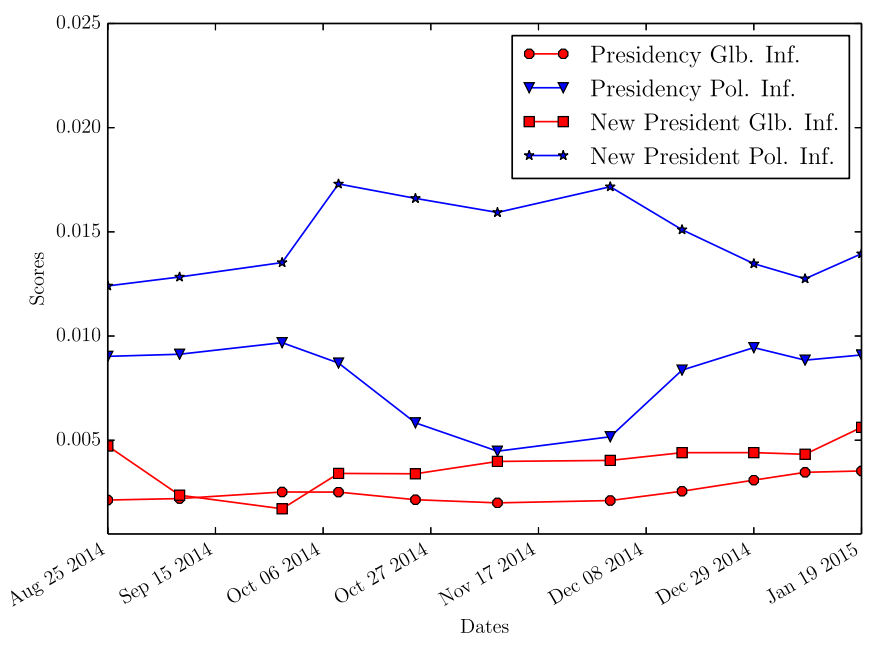

Fig. 2. Past global and topic-based (politics) influence scores of the presidency of the Republic of Turkey and the newly elected president.

scores (calculated on true snapshots) history of users, which are selected by our algorithm as one of the most important users that should be probed. These are the official accounts of the presidency of the Republic of Turkey and the newly elected president. Besides their high impact, we observe that their influence also varies significantly over time, which further justifies the need to probe these accounts frequently. A reason of the variation in influence score is that the time period shown in the figure matches with the elections for the Presidency (10 August 2014). After becoming the new president, the president account's global influence has further increased. During this period, it is always selected as a top user to be probed by our proposed approach. This is intuitive, as it is a popular account with changing influence scores over time. We can also observe the impact of presidential change on the presidency account. During this change, its global score slightly decreases and then starts to increase.

Topic-Based Influence Score. The system calculates topicbased influence scores representing user activity and impact on a specific topic. We perform semantic analysis on user tweets by taking re-tweets and favorite counts into consideration as well. A re-tweet is a re-posting of someone else's tweet, which helps users quickly share a tweet that they are influenced by or like. A favorite is another feature that represents influence relation between users, wherein a user can mark a tweet as a favorite. These two features help estimate the influence of an individual tweet. Since Twitter is a microblogging platform, users are generally tweeting on specific topics. While many tweets are mostly conversational and reflect self- information [5], [6], some are being used for information sharing, which is important in harvesting knowledge. RTs and FAVs are effective in separating relevant and irrelevant tweets. Accordingly, we use them in our topic weight analysis to estimate influence of a tweet on a specific topic.

Topic-based network construction process consists of three main phases: a) keyword extraction on tweets, $b$ ) correlation of keywords with topic dictionaries, and c) weight calculation.

In the first phase, keywords are extracted from tweets by using information retrieval techniques, including word stemming and stop word elimination. The output from this phase is a keyword analyzed tweet corpus for each 
individual user and the related histogram which captures the frequencies of the related keywords $(K)$. These corpora are further analyzed in the second phase.

We have created a keyword dictionary $\left(D_{j}\right)$ for each topic $\left(C_{j}\right)$, in order to score tweets against topics. Each dictionary contains approximately 90 to 130 words. In order to create a dictionary for a topic, we first compose a representative word list for the topic. We then divide these words into groups according to context similarity and assign weights to word groups within a scale (such as in range $[1 \ldots 10]$ ). Context similarity can be determined by a domain expert utilizing knowledge about the taxonomy. Similarly, we repeat the process for all topics. As part of each dictionary, we have assigned normalized weights to words, representing their topic relevance. In the second phase, using the weights from the dictionaries and the users' keyword histograms, we obtain the normalized raw topic scores of users for each one of the topics.

In the third phase, we calculate a value called the RTFAV total for each user, which is the summation of the number of re-tweets and favorites received by a user's tweets. We then multiply the normalized raw topic score by the RTFAV total of the user, in order to find the number of RTFAVs the user gets on a topic of interest. The final normalized results are used as the in-edge weights of the users on each topic, when forming the topic-based network.

Once the topic-based network construction is complete, we execute the weighted PageRank [4] (WPR) algorithm which also considers the importance of the incoming and outgoing edges in the distribution of the rank scores. The resulting weighted PageRank values of users, denoted by $W P R_{v}(t)$ at time $t$ for $v \in G_{t}$, is assigned as their topic-based influence scores.

Due to the nature of the PageRank algorithm, some of the globally influential users also turn out to be highly influential for most or all of the topics. These users have a lot of followers and they are also followed by some of the influential accounts of the specific topics, which cause them to score high for topic-based analysis as well. Therefore, they can get high topic-based influence scores even if they do not actively tweet about the topic itself. To eliminate this effect, we apply one more level of filtering to remove these globally effective accounts from the topic-sensitive influence lists. In particular, if the number of tweets a user posted that are related with the topic at hand is less than a predefined percentage, e.g., percent $40,{ }^{6}$ of the total number of tweets posted by the user, then the user is discarded for that topic's score list. This filtering process significantly reduces the noise level in the analysis.

As a result, for each topic, we construct a weighted network in which an edge $((u, v))$ represents the amount of topic-specific influence a user $(v)$ has on a follower user $(u)$. Thus, the results of weighted PageRank algorithm gives us the overall topic-influence scores on the network.

Fig. 2 also shows the topic-based score history of the official account of the presidency of the Republic of Turkey and the newly elected president. We can see from the figure that the change in the topic-based scores are more dramatic

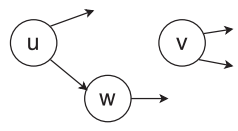

(a) Previous state of the network before new edge.

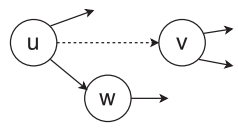

(b) Current state of the network after new edge

Fig. 3. A sample network for analysis.

compared to the global scores. This is intuitive, as the topicsensitive scores are depending on users' tweets and sharing statistics. A user might be very active on some weeks about a specific topic such that her influence on the topic might increase dramatically. Likewise, when she posts something important, it might achieve high sharing rates. On the other hand, when she just posts regular tweets which are not shared, her influence on the topic might decrease quickly.

\section{Dynamic Data Fetching}

In this section, we introduce our algorithms for probing dynamic social networks. In order to efficiently determine a subset of vertices to probe, we develop heuristics for both dynamic network fetching and dynamic tweet fetching problems given in Section 2.

Since we have chosen the PageRank score as the indicator of influence in a social network, we analyze its change as the network evolves. PageRank value of a specific vertex $v$ is given as follows:

$$
P R(v)=\alpha \sum_{\forall(u, v) \in E_{\text {in }}(v)} \frac{P R(u)}{\left|E_{\text {out }}(u)\right|}+\frac{1-\alpha}{n},
$$

where $P R(v)$ denotes the PageRank value, $E_{\text {in }}(v)$ denotes the in-edge set, and $E_{\text {out }}(v)$ denotes the out-edge set for $v$.

Fig. 3 shows an example network, which will be used to demonstrate the effects of network changes on PageRank values.

Assume that an edge $(u, v)$ is added to the state in Fig. 3a due to the evolving nature of the network. The resulting current state is shown in Fig. 3b. Here, we analyze the effect of this addition on the PageRank values of the out neighbors of $u$. We see that the PageRank value of $v$ is as follows per Eq. (3):

$$
\begin{aligned}
P R^{\text {new }}(v) & =\alpha\left(\sum_{\forall(i, v) \in E_{\text {in }}(v)} \frac{P R(i)}{\left|E_{\text {out }}(i)\right|}+\frac{P R(u)}{\left|E_{\text {out }}(u)\right|+1}\right)+\frac{1-\alpha}{n} \\
& =P R(v)+\alpha \frac{P R(u)}{\left|E_{\text {out }}(u)\right|+1} .
\end{aligned}
$$

We can easily extend this analysis to multiple new edges since the total effect will be a superposition of the effect of the new individual in-edges of vertex $v$

$$
P R^{\text {new }}(v)=P R(v)+\alpha \sum_{\forall(u, v) \in E_{\text {in }}^{\text {new }}(v)} \frac{P R(u)}{\left|E_{\text {out }}(u)\right|+1} .
$$


PageRank values of out neighbors of $u$ other than $v$, such as $w$, are impacted as follows:

$$
\begin{aligned}
P R(w) & =\alpha\left(\sum_{\forall(i, w) \in E_{\text {in }}(w) \backslash(u, w)} \frac{P R(i)}{\left|E_{\text {out }}(i)\right|}+\frac{P R(u)}{\left|E_{\text {out }}(u)\right|}\right)+\frac{1-\alpha}{n} \\
P R^{\text {new }}(w) & =\alpha\left(\sum_{\forall(i, w) \in E_{\text {in }}(w) \backslash(u, w)} \frac{P R(i)}{\left|E_{\text {out }}(i)\right|}+\frac{P R(u)}{\left|E_{\text {out }}(u)\right|+1}\right)+\frac{1-\alpha}{n} \\
P R^{\text {new }}(w) & =P R(w)-\alpha \frac{P R(u)}{\left|E_{\text {out }}(u)\right| \cdot\left(\left|E_{\text {out }}(u)\right|+1\right)} .
\end{aligned}
$$

These effects are the immediate responses on the vertices that are considered. These residual PageRanks will ripple out to all the vertices in all the paths from $v$ and $w$ in each iteration of the PageRank algorithm. But the effect will decease as the residuals will be divided by the number of outgoing edges for each vertex visited. We will analyze the effects of the first iteration of the algorithm to simplify the problem and to get a general feel of the change in PageRank values. Considering expected value of $\overline{E_{\text {out }}}=E\left[\left|E_{\text {out }}(u)\right|\right]$ as the average out-degree for vertices, the differential PageRanks are given as follows:

$$
\begin{aligned}
& \nabla P R(v)=\alpha \frac{P R(u)}{\overline{E_{\text {out }}}} \\
& \nabla P R(w)=-\alpha \frac{P R(u)}{\overline{E_{\text {out }}}} .
\end{aligned}
$$

We can see from Eqs. (4) and (5) that we should select the vertices, say $u$, with the following properties for accurate $G_{t}^{\prime}$ and $P R_{u}^{\prime}(t)$ estimations:

- $\quad$ vertices with high PageRank values $(P R(u))$;

- vertices whose PageRank values change over time;

- vertices with high out-degrees $\left(E_{\text {out }}(u)\right)$;

- vertices whose out-degrees change over time.

PageRank, when computed until the values converge in steady state, considers both incoming and outgoing edges. The parameters related to out-degree values are intrinsically taken into account when PageRank is computed. Hence, in our dynamic fetching approach, we focus only on PageRank values and their changes to cover all the cases listed above.

Based on these observations, we will define a utility function that incorporates the above findings. We will find the vertices that maximize this utility function, which will be probed and used to estimate the influence scores of the evolving network. We analyze two sub-problems of the general case specific for our application: network fetching and tweet fetching. These sub-problems and the solutions will be addressed in the subsequent sections.

\subsection{Dynamic Network Fetching Using Influence Past}

We aim to probe a subset, $U_{t}^{N}$, update the edges incident on vertices in $U_{t}^{N}$ to form $G_{t}^{\prime}$, and calculate PageRank values $P R_{v}^{\prime}(t), \forall v \in G_{t}$. In order to determine this subset, we use a time series of past PageRank values for a vertex $v$, named the influence past of $v$. Formally, we have $I P_{v}=\left[\ldots, P R_{v}^{\prime}(t-2), P R_{v}^{\prime}(t-1)\right]$.
In our strategy for determining $U_{t}^{N}$, we consider the vertices whose PageRank values change considerably over time. We first explored building time-series models over sequences of scores to forecast their future values. There are some well-known methodologies in the literature for forecasting using this kind of time-series data, such as ARIMA models [7]. However, these models typically require much longer sequences for accurate predictions. Therefore, in order to quantify this change for a vertex $v$, we calculate the standard deviation of the time series $I P_{v}$, that is

$$
\text { Change }_{v}=\sigma_{I P_{v}}=\sqrt{\operatorname{Var}\left(P R_{v}^{\prime}\right)} .
$$

Choosing the best vertices to probe can be performed by calculating a score that is a linear combination of the PageRank value and the change in PageRank values, as given in Eq. (7). Here, $\theta$ parameter balances the importance of the two aspects. We assume that influence past that contains at least two data points is available for every user, in order to calculate the score changes

$$
\operatorname{Score}(v)=(1-\theta) P R_{v}^{\prime}(t-1)+\theta \text { Change }_{v} .
$$

After the selection of the users with respect to the ranking of $\operatorname{Score}(v)$, we probe their current relations and form $G_{t}^{\prime}$.

Round-Robin and Change Probing. Change Probing could cause the system to focus on a particular portion of the network and may discard the changes developing in other parts. This is because the probing scores of some vertices will be stale and as a result these vertices may consistently rank below the top- $k$, despite changes in their real scores. This bias could end up accumulating errors in the influence scores of these vertices and start to have an impact on the entire network. Therefore, we propose to use Change Probing together with Round-Robin Probing, in which users are probed in a random order with equal frequency. In this way, we aim to probe every vertex at least once within a specific period $\operatorname{Prr}$ s.t. $\operatorname{Prr} \leq\left|V_{t}\right| * P /((1-\beta) * k)$. RoundRobin Change algorithm probes some portion of the network randomly and marks all probed users. Thus, any probed users are not probed randomly again, until all users are probed at least once within $P$. In this method, we control the balance between change versus random selection by using a parameter $\beta \in[0,1]$. In particular, we choose $\beta * k$ users to probe with Change Probing and $(1-\beta) * k$ users with Round-Robin Probing.

Network Inference. Since we are able to fetch data only for a limited number of users, there is a high probability that other users in the network have changed their connections as well. To take these possible changes into account, we have incorporated link prediction into our solution. Link prediction algorithms assign a score to a potential new edge $(u, v)$ based on the neighbors of its incident vertices, denoted as $\Gamma_{u}$ and $\Gamma_{v}$. The basic idea behind these scores is that the two vertices $u$ and $v$ are more likely to connect via an edge if $\Gamma_{u}$ and $\Gamma_{v}$ are similar, which is intuitive. Considering social networks, two people are likely to be friends if they have a lot of common friends. There are different scores used in the literature, including the common neighbors, Jaccard's coefficient, Adamic/Adar, and Resource 
Allocation Index (RA). We use RA as part of our approach, since it was found successful on a variety of experimental studies on real-life networks [8]. One could also adopt more advanced prediction algorithms such as [9], in order the increase effectiveness of this approach.

RA is founded on the resource allocation dynamics of complex networks and gives more weight to common neighbors that have low degree. For an edge $(u, v)$ between any two vertices $u$ and $v$, RA is defined as follows:

$$
R A_{u, v}=\sum_{w \in \Gamma_{u} \bigcap \Gamma_{v}} \frac{1}{\operatorname{degree}(w)},
$$

where $\Gamma_{v}$ is the neighbors of $v$.

The $R A$ score, $R A_{u, v}$ for the edge $(u, v)$, is proportional to the probability of an edge being formed between the vertices $u$ and $v$ in the future. Based on this, we rank all the calculated $R A$ scores. Since the edges in our network are not defined probabilistically and are defined deterministically as existent or non-existent, we need to determine how many of these scored edges should be selected. Therefore, we define a growth rate, $E_{q}$, which is the average change in the number of edges $(|E|)$ between snapshots of the network after excluding the changes due to $U_{t}^{N}$. After calculating RA scores for all possible new edges, we choose $E_{g}$ edges with the highest scores. Using this method, we add new connections to the current graph, to finally have the estimated graph $G_{t}^{\prime}$. The pseudo code of the network inference based probing algorithm we use to select $k$ vertices to probe is given in Algorithm 1.

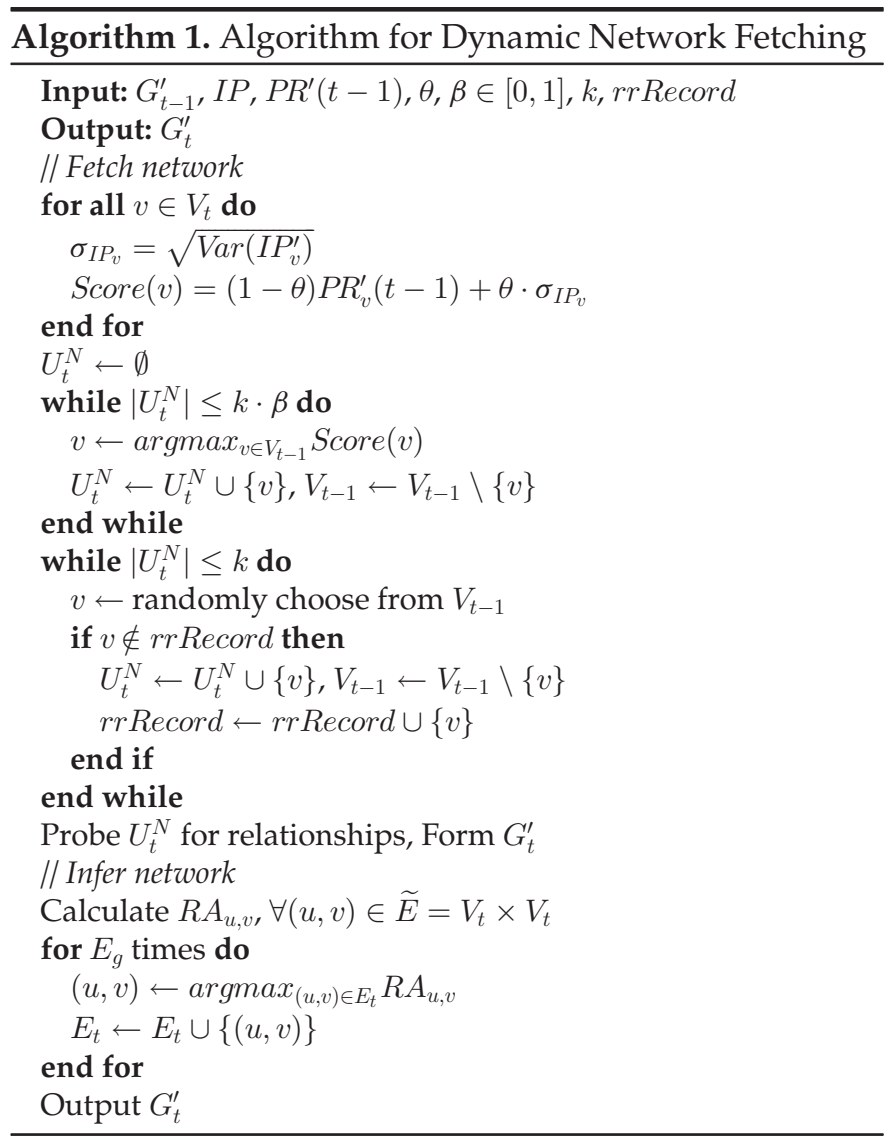

\subsection{Dynamic Tweet Fetching Using Topic-Based Influence Past}

Our dynamic tweet fetching solution makes use of the weighted PageRank values and comprises of two steps. First, we infer the evolving relationships of the network using the methods explained earlier in the previous section. This way we can track and estimate the changing relationships. Second, we select a subset of users to fetch their tweet data. Specifically, we aim to probe a subset, $U_{t}^{T}$, collect their tweets, and update the edge weights for the users in $U_{t}^{T}$; all in order to form $W G_{t}^{j^{\prime}}$ for a given topic $C_{j}$. We then compute weighted PageRank values to find $W P R_{v}^{j^{\prime}}(t), \forall v \in W G_{t}^{j}$ for a given topic $C_{j}$. To select the subset of users in $U_{t}^{T}$, we use a time series of the past weighted PageRank values, named the topic-based influence past of $v$. Formally, we have $T I P_{v}=$ $\left[\ldots, W P R_{v}^{j^{\prime}}(t-2), W P R_{v_{i}}^{j^{\prime}}(t-1)\right]$. This is performed independently for all topics of interest, $\left\{C_{j}\right\}$.

There are two different approaches we employ to track the topic-based influence scores:

- Use the global network parameters for network fetching and the topic-sensitive network parameters for tweet fetching. This is named as the $G-W G$ method, where global $G_{t}$ is used for network fetching, and topic-sensitive $W G_{t}$ is used for tweet fetching.

- Use the topic-sensitive network parameters for both network and tweet fetching. This is named as the $W G-W G$ method.

The first approach, $G-W G$, is useful for cases where globally influential users are tracked, but with minimal additional resources, topic-based influential users are to be determined as well. This might be the only viable option if the bandwidth is not enough for selecting and updating the vertices separately for each topic, especially if the number of topics is high. For the second approach, that is $W G-W G$, we construct separate networks $W G^{j}$ for each topic and evolve them separately. We update each network at the end of a probing period, using the new tweets fetched to track the most influential vertices for each topic $C_{j}$. The high-level algorithm for the $G-W G$ method is given in Algorithm 2. The algorithm for $W G-W G$ is very similar, and is omitted for brevity.

\section{EXPERIMENTS AND RESULTS}

In this section, we present the experimental setup and the results of our evaluation of the proposed algorithms. We also present experiments analyzing the sensitivity of the parameters used.

\subsection{Data Sets}

We collected data using the public Twitter API, as described in Section 3. These API calls are restricted by rate limit windows. These windows represent 15 minute intervals and the allowed number of calls within each window can vary with respect to the call type. Our system makes three different calls, a) "GET followers/ids", which returns user IDs for every user following the specified user, $b$ ) "GET friends / ids", which returns user IDs for every user the specified user is following, and c) "GET statuses/user_timeline", which returns the most recent Tweets posted by the specified user. For the first two call type, we are allowed to make 15 calls 

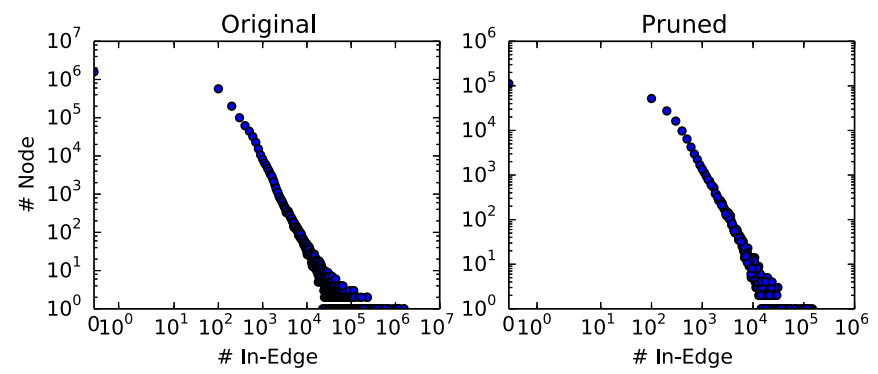

Fig. 4. In-edge distributions of the original network (on the left) and the pruned network (on the right).

per window. Every call can return up to $5 \mathrm{~K}$ followers/ friends. For the users who have more than $5 \mathrm{~K}$ followers/ friends, we have to make multiple calls, accordingly. For the third type, we are allowed to make 180 calls per window. Each call can return 200 tweets of the queried user. Details of the calls are also presented in Section 2 with the accompanying analysis.

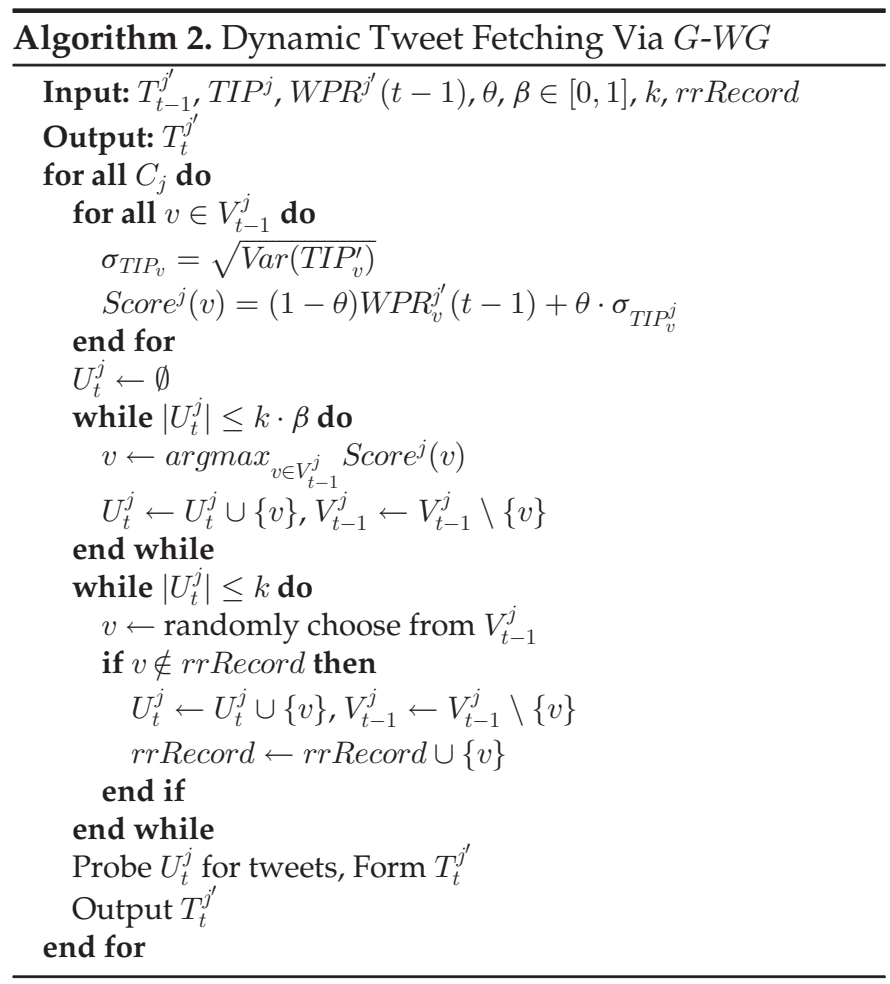

We collected the network between the end of August 2014 and the beginning of January 2015, with a period of 1520 days. As a result, we have obtained 11 snapshots of the Turkish users' network with progressing timestamps. We collected the relations of 2.8 million users, which amounts to a total of 310 million edges on average. Users are recrawled for each snapshot so that snapshots contain exact information with respect to the network. We took the first snapshot as the initial network to calculate the probing scores (see Eq. (7)) and the rest of the snapshots were used as ground truth for the evaluation of the probing algorithms. For the topic-based influence estimation, we also collected the tweets of our seed users in the same period. We constructed a dataset formed of 11 snapshots containing 5.5 billion tweets in total. We take the first snapshot as the initial tweet set as in the case of the relationship network analysis.

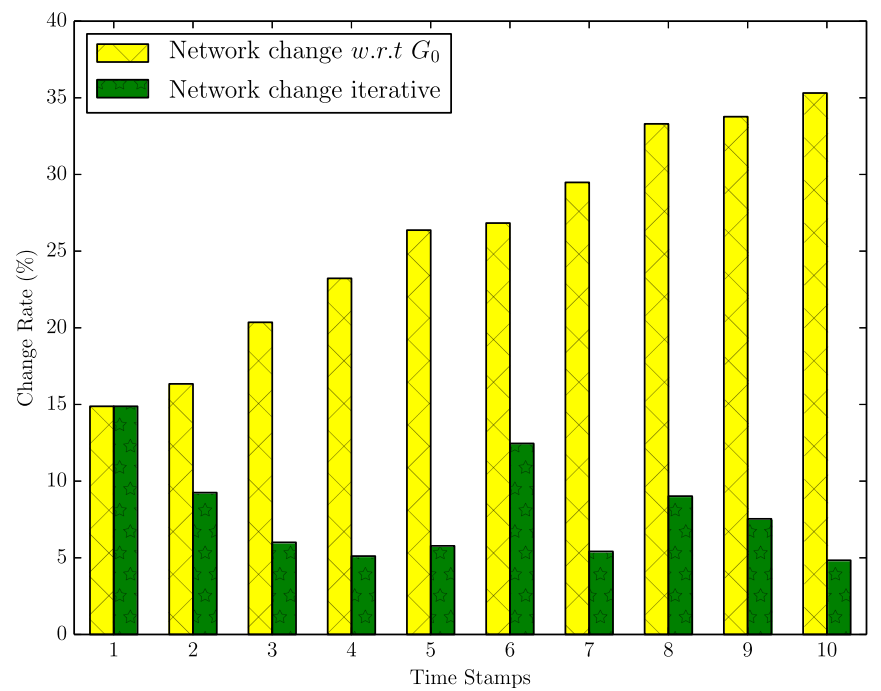

Fig. 5. Change rate of the network over each iteration w.r.t the previous one and w.r.t. the original one.

From this data, we built up the topic weighted networks and calculated probing scores (see Eq. (7)), accordingly.

In our probe simulation module, we fetch the connections of the users we have selected for probing, from the real network $G_{t}$ at time $t$. We then update these connections (adding new ones and deleting old ones) on the previously observed network $G_{t-1}^{\prime}$ at time $t-1$, in order to obtain the estimated network $G_{t}^{\prime}$ at time $t$. Finally, we compare the influence estimation results from the observed network $G_{t}^{\prime}$ with the ones from the real network $G_{t}$. Same procedure is also applied for the tweet sets.

In order to include extensive number of experiments in our evaluation, we focused on the top $250 \mathrm{~K}$ influential users and restricted the network on which the scores are computed to the network formed by these users.

Fig. 4 shows the in-edge distribution of the original and the pruned network. Both follow a power-law distribution. Impact of the pruning process on the network structure seems to be minimal and has not created any anomalies in the analysis. We also pruned the tweet list according to the same top $250 \mathrm{~K}$ influential users, which reduced the total size of the tweet sets to $200 \mathrm{M}$. Fig. 5 shows how much the network has changed over each iteration with respect to the previous snapshot $\left(\frac{\left|E_{t} \backslash E_{t-1}\right|}{\left|E_{t-1}\right|}\right)$ and with respect to the original one $\left(\frac{\left|E_{t} \backslash E_{0}\right|}{\left|E_{0}\right|}\right)$. Here, change w.r.t. previous snapshots is defined in order to have an insight about the experimental data and it cannot be compared with the experimental results of the any probing strategy. It represents the case where exact snapshots of the network exist locally, which is not the case in a real-world scenario. In a probing scenario where the exact network is not available, network error is expected to increase, as we are continuously building on top of the previous partial network which also contains some amount of error. Therefore, iterative change w.r.t. original network better matches a real-world scenario.

\subsection{Evaluation of Dynamic Network Fetching}

We have implemented several algorithms to compare the performance of the proposed techniques. The details of the algorithms used are given as follows: 
NoProbe and Random Probing. These are two baseline algorithms. NoProbe algorithm assumes that the network does not change over time and uses the fully observed network at time $t=0$ for all time points without performing any probing. It represents the worst case scenario for dynamic network fetching. The second baseline algorithm is Random Probing algorithm which randomly chooses $k$ users to probe with uniform probability. In the experiments, this baseline method is run 10 times and the average values of these runs are used in the evaluation.

Indegree Probing. This is our third baseline algorithm that uses a very similar idea to our proposed technique from Eq. (7). This baseline method utilizes the same formula with one change, instead of using PageRank values it uses the indegree values of the users $(\operatorname{Score}(v)=$ $\left.(1-\theta) D e g_{v}^{\prime}(t-1)+\theta \sigma_{I P_{v}^{D e g}}\right)$.

MaxG. As described in [10], users are probed with a probability proportional to the "performance gap", which is defined as the predicted difference between the results of the approximate solution and the real solution. Briefly, the method incrementally probes users which will bring the largest difference in the results. It assumes that the influence of a specific user is related to the output of the degree discount heuristic. Although their influence determination function is different than ours, we use the MaxG algorithm for performance evaluation of our proposed algorithms.

Priority Probing. As described in [11], this algorithm chooses users to probe according to a value proportional to their priorities. Priority of a node is defined as the value of its PageRank score. For every iteration of the method, if a node is not probed, the current PageRank value is added to its priority and if the node is probed, its priority is reset to 0 .

Change Probing. This is our first proposed method, which chooses $k$ users to probe with value proportional to their scores, as computed by Eq. (7). The network is then constructed via Algorithm 1.

RRCh Probing. This is our second proposed method, which chooses $\beta \cdot k$ users to probe with Change Probing and $(1-\beta) \cdot k$ users with Round-Robin Probing. When $\theta=0$ in Eq. (7) for the Change Probing part, the method becomes similar to [11]. The difference is that Priority Probing increases the probe possibility of a node by its PageRank value in every step if it is not probed, so that at some point the probe possibility becomes 1 .

We evaluate performance by comparing the quality of the influential users found by each approach with that of the ideal case. For this purpose, we use two different evaluation measures:

- Jaccard similarity between the correct and estimated top- $k$ most influential users lists.

- The mean squared error (Eq. (9)) of the PageRank scores. The reported values with respect to the probing capacities of MSE are the average values of all 11 snapshots. The values with respect to time are the average values of different probing capacities. Additionally, standard deviations of the values are also reported in the discussions

$M S E=\sqrt{\frac{1}{\left|V_{t} \cap V_{t}^{\prime}\right|} \sum_{\forall v \in V_{t}^{\prime} \cap V_{t}}\left(P R_{t}^{\prime}(v)-P R_{t}(v)\right)^{2} .}$

\subsection{Evaluation of Dynamic Tweet Fetching}

We evaluate the performance of the proposed tweet fetching technique with two baselines algorithms, namely NoProbe and Random Probing. The details of these baselines are given below:

NoProbe. This algorithm assumes that the tweet set does not change over time and use the fully observed tweet set at time $t=0$ for all time points without any probing. This method represents the worst case scenario for the dynamic tweet fetching problem.

Random Probing. This algorithm randomly chooses $k$ users to collect tweets with uniform probability at each time step.

RRCh Probing. This is the algorithm we proposed, which greedily chooses $k$ users to collect tweets with value proportional to their scores describe in Eq. (7). Differently from the network fetching method, scores are calculated by using $W P R_{v}^{j}$ for the topic $C_{j}$, instead of $P R_{v}$.

\subsection{Experimental Results and Discussion}

This section compares and discusses the performance of the proposed network and tweet probing methods with the state-of-the-art and baseline methods using experiments executed on real datasets. We also provide an empirical interpretation of the calculated topic-based influence scores.

\subsubsection{Experimental Setup}

As indicated by Eqs. (1) and (2), given the resource limits permitted by the service providers, one cannot probe a significant portion of the network. We have executed our experiments with different probing capacities and used $0.001,0.01,0.1$ and 1 percent of the network as the size of the probe set. For the analysis of the effect of the $\theta$ parameter used in Change Probing, we set: a) $\theta=0$, meaning PageRank proportional scores are used; $b$ ) $\theta=0.5$, meaning equally weighted PageRank and influence past scores are used; c) $\theta=1$, meaning only influence past scores are used. For the RRCh algorithm we tested the ratio parameter $\beta$ with three values, which control the fraction of vertices proved via random selection: $0.4,0.6$, and 0.8 .

\subsubsection{Change Probing Performance w.r.t. $\theta$}

Fig. 6 depicts the performance of Change Probing algorithm for the average Jaccard similarity and MSE measures. As expected, Change Probing algorithm significantly outperforms NoProbe algorithm. For the optimization of the $\theta$ parameter, we test Change Probing algorithm under three different $\theta$ configurations:

- Using the MSE measure, $\theta=0.5$ setting performs 8 percent better than $\theta=0$ setting and 19 percent better than $\theta=1$ setting. Overall, it performs 83 percent better than NoProbe.

- Using the Jaccard distance measure, $\theta=0.5$ setting is 3 percent better than $\theta=0$ setting and 5 percent better than $\theta=1$ setting. In the overall case, $\theta=0.5$ outperforms NoProbe by 43 percent. We also note that as the probing capacity increases, performance of the Change Probing algorithm becomes less dependent on the setting of $\theta$. 


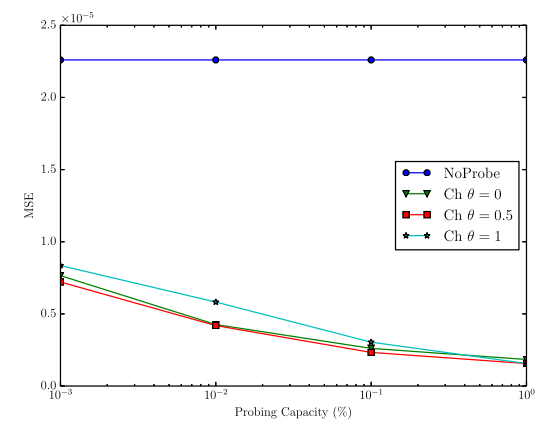

(a) Average MSE for all snapshots.
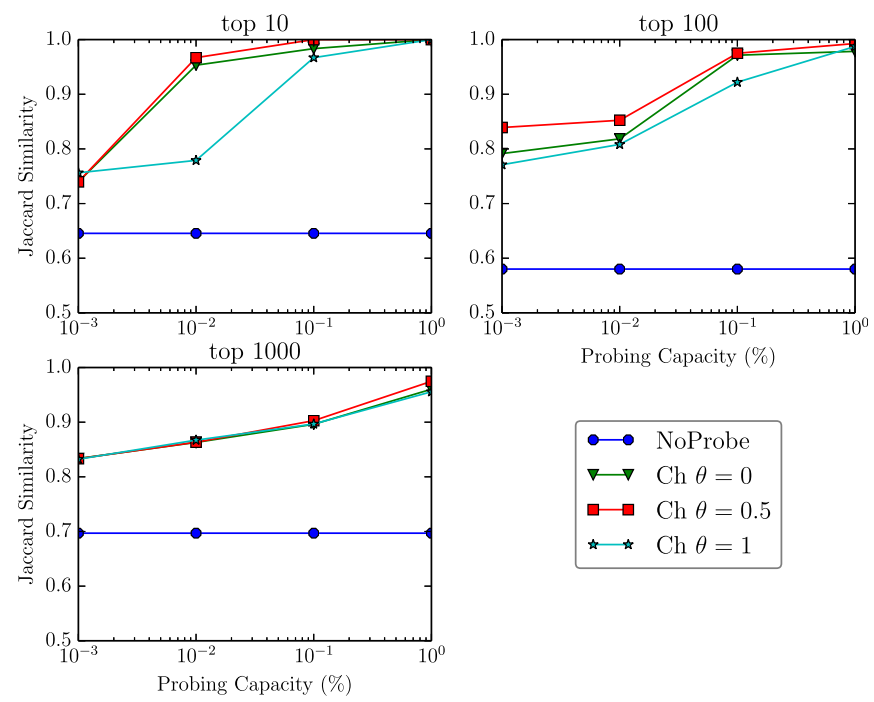

(b) Average Jaccard similarity for all snapshots.

Fig. 6. Performance of change probing w.r.t. $\theta$.

We also illustrate the change in error as the network evolves, in order to see how the performance of different algorithms are affected as the seed network data ages. Figs. $7 \mathrm{a}$ and $7 \mathrm{~b}^{7}$ show the performance of Change Probing as a function of time for the mean squared error (MSE) and Jaccard similarity measures, respectively. We observe that NoProbe has an increasing error as time passes. Change Probing gives a more robust and stable performance with respect to time. As the number of past influence points increases, the algorithm can estimate the influence variability of the users more accurately, which compensates the deteriorating effect of aging of the baseline network data. Since $\theta=0.5$ outperforms the other cases, we use $\theta=0.5$ configuration in the subsequent experiments with other algorithms. We also note that $y$-axis contains relatively small values because the PageRank values are normalized. We have assumed NoProbe algorithm as the reference point for normalization.

\subsubsection{RRCh Probing Performance w.r.t. $\beta$}

Fig. 8 shows the performance results for the Round-Robin Change (RRCh) Probing algorithm under different roundrobin ratios. We use the Change Probing algorithm (with $\theta=0.5$ setting) as the baseline reference point.

We observe that the RRCh algorithm performs poorly for small probing capacities, such as 0.001 and 0.01 percent.

7. Jaccard similarity reports the average values of all three probing capacity settings.

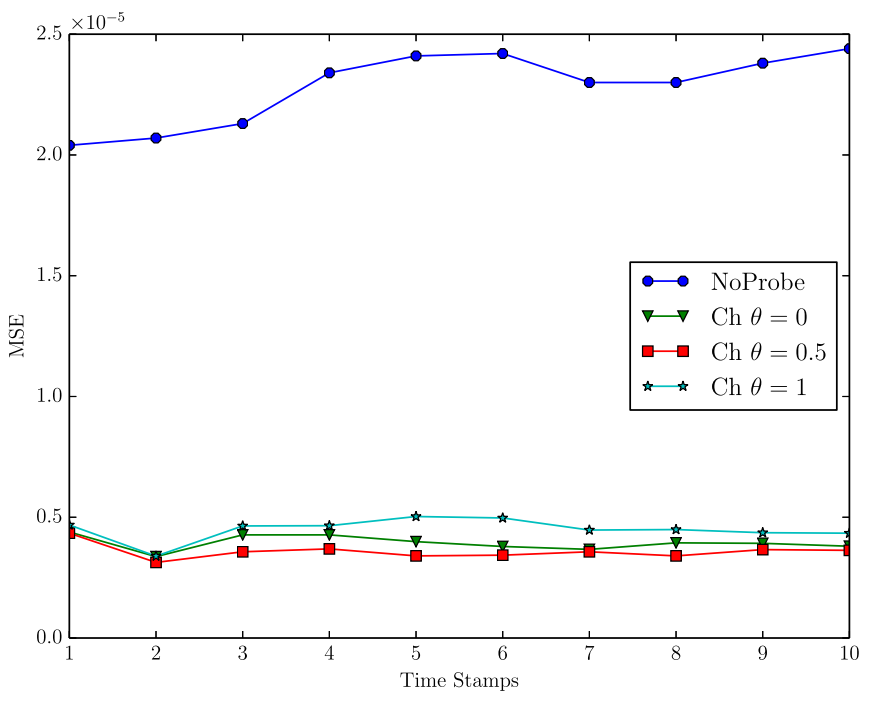

(a) Average MSE for all probing capacities.

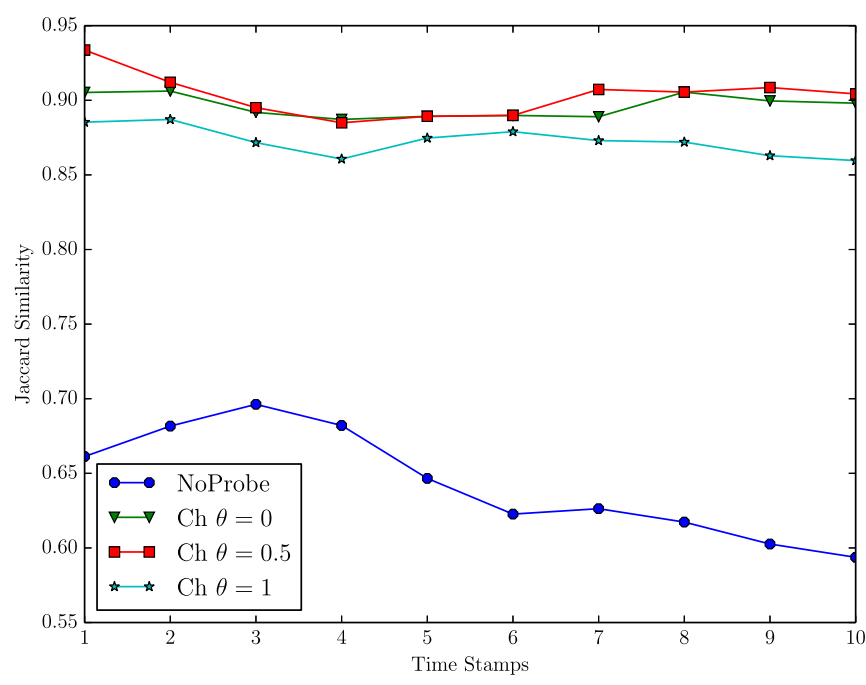

(b) Average Jaccard similarity for all probing capacities.

Fig. 7. Performance of change probing as a function of time.

Randomness impacts the performance more with smaller number of probed users, since we are not able to probe the influential users with great influential power, thus lowering the performance. For MSE, $\beta=0.8$ configuration performs 7 percent better than $\beta=0.6$ and 12 percent better than $\beta=0.4$. For the Jaccard similarity measure, it is 2 percent better than $\beta=0.6$ and 7 percent better than $\beta=0.4$. Although, it performs worse than Change Probing in the short term, it reaches the performance of Change Probing in the long term, as show in in Figs. 9a and 9b. Moreover, it guarantees the probing of every node within a time frame, preventing the system to focus on only a limited section of the network and missing other regional changes that might accumulate and start to affect the network in the global sense. We would have seen this phenomenon more explicitly if the number of snapshots were larger, which was the case in [10]. The results are slightly better when the ratio is set to $\beta=0.8$. Therefore, we choose to use this algorithm (with $\theta=0.5$ and $\beta=0.8$ configurations) instead of Change Probing for the comparison with others in the following sections.

Fig. 10 shows both the percentages of edges that were not present in the the true network but were assumed to be 


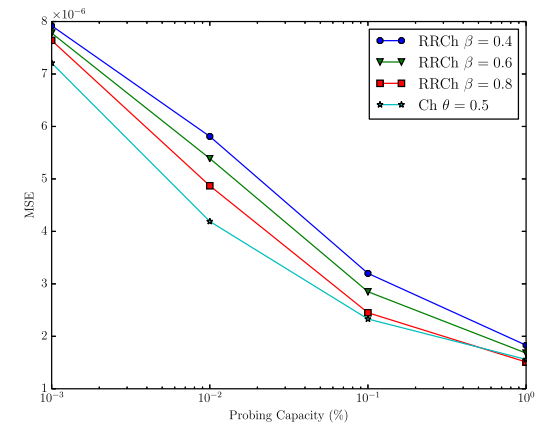

(a) Average MSE for all snapshots.
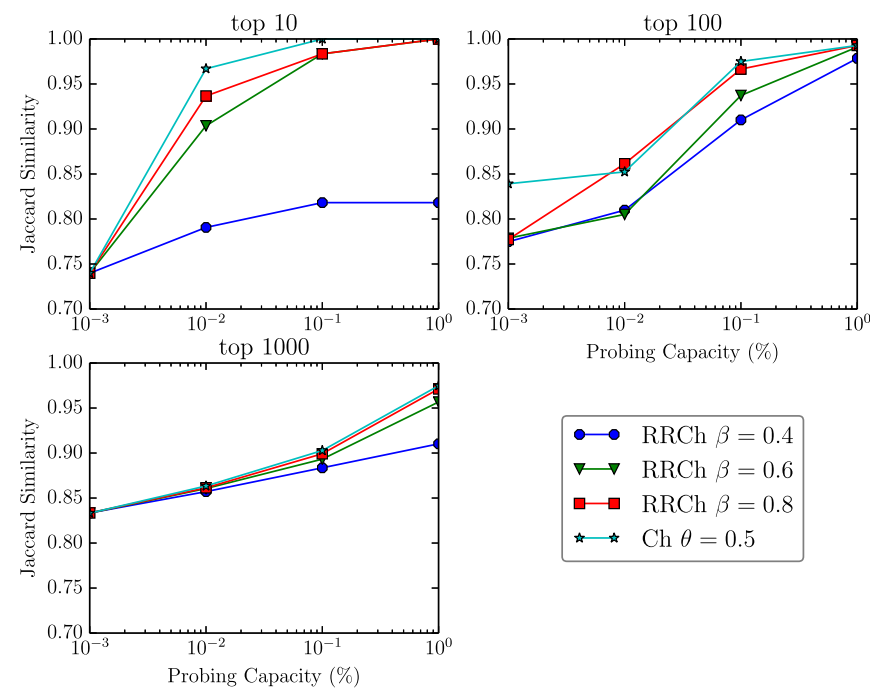

(b) Average Jaccard similarity for all snapshots.

Fig. 8. Performance of RRCh w.r.t. $\beta$.

present by the algorithm (false positives) and the percentages of edges that were present in the true network but were not captured by the algorithm (false negatives). The findings indicate that the proposed technique is doing a good job at capturing the structure of the network by having on average 12 percent false positives and 6 percent false negatives rates for all snapshots.

\subsubsection{Comparison with the State-of-the-Art}

Fig. 11 compares the performance of RRCh method (with $\theta=0.5$ and $\beta=0.8$ settings) against the baselines and the state-of-the-art methods from the literature. RRCh achieves better results for all performance measures used for comparison in our paper. It reduces MSE by 21 percent (see Fig. 11a) when compared to Priority Probing, 41 percent when compared to Indegree Probing and 49 percent when compared to the MaxG method. Priority Probing suffers especially for low probing capacities, since the priority of a user is set to 0 after probing. A probed user can regain its priority very late in the process, which prevents it to track quick changes in the scores of the highly influential users. Therefore, after probing an important user in terms of influence, that user is not being probed for some time, even if the influence of the user is changing very fast. RRCh always probes $\beta$ portion of the users according to their influence impact and change over time, so that the important users are in the probe set at each step.

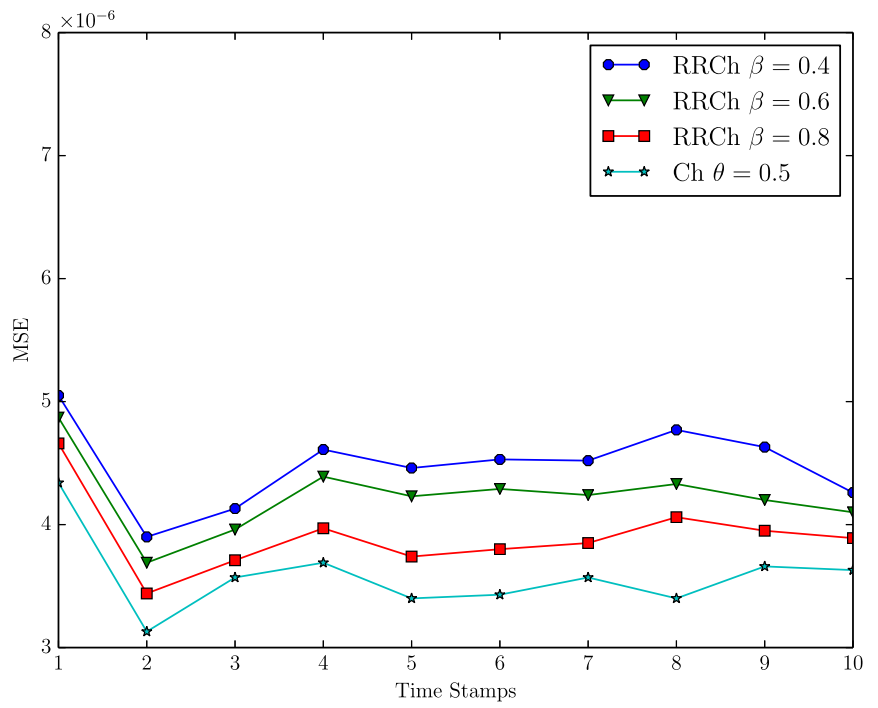

(a) Average MSE for all probing capacities.

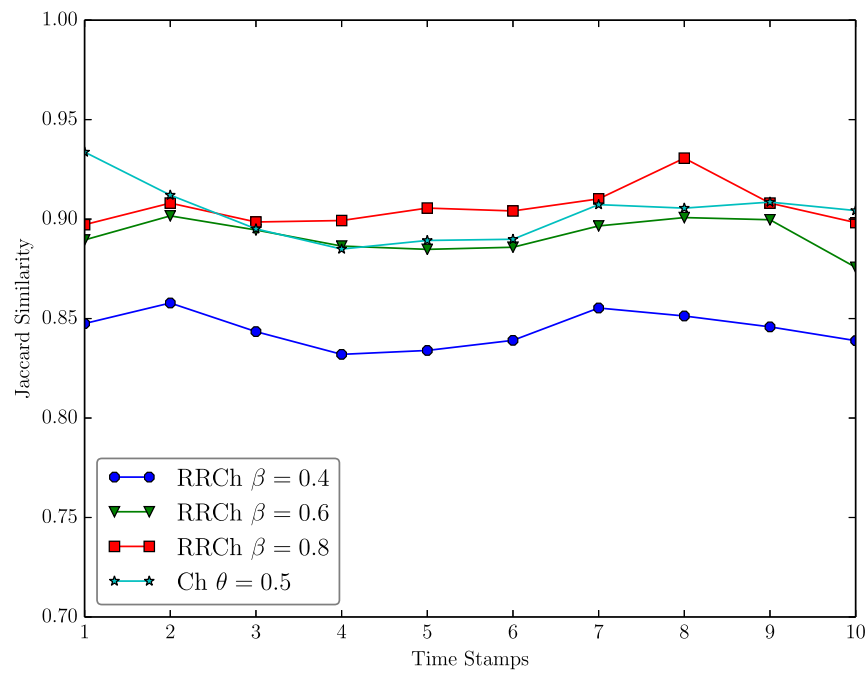

(b) Average Jaccard similarity for all probing capacities.

Fig. 9. Performance of RRCh as a function of time.

Overall, our proposed method gives 80 percent higher performance than the NoProbe and Random Probing algorithms for the MSE measure. As seen in Fig. 11b, RRCh shows better results for the top-k set similarities as well. It is 5 percent better than Priority Probing, 7 percent better than Indegree Probing and 11 percent better than MaxG method on average. RR Change performs 35 percent better against baselines when Jaccard similarity is considered. Since it also considers the change in the influence over time, it is also able to preserve its accuracy while the performance of other methods degrade over time (see Figs. 12a and 12b).

As mentioned before, in real-world scenarios one might not be interested in the exact rank of the influential users but instead might select top-k users and evaluate them by personal observation, because the ranking may not be so accurate. Yet, we also compared the probing techniques against a rank-aware similarity measure. Fig. 13 shows the performance of alternative probing strategies based on the Kendall Tau-b metric. The results are the average values from all of the snapshots. RRCh gives 73 percent higher performance than Random probing, 58 percent higher than 


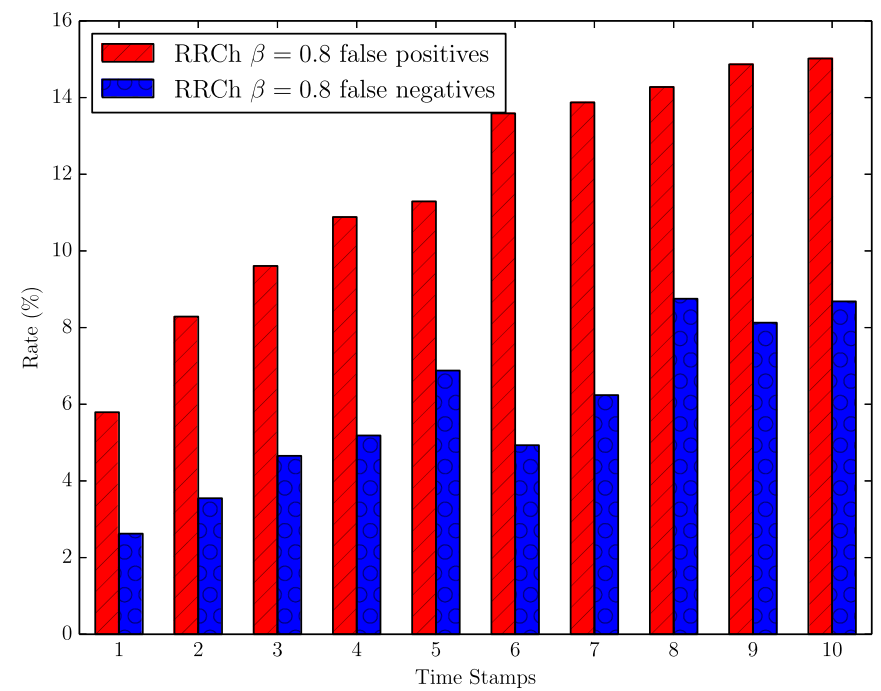

Fig. 10. False positives and false negatives rates for every snapshot in time.

Indegree Probing, 47 percent higher than MaxG method and 40 percent higher than Priority Probing.

\subsubsection{Evaluation of the Network Inference Method}

To assess the prediction quality of the link prediction algorithm, we plotted the histogram of the edges proposed by RA index that has really occurred in the real network. This

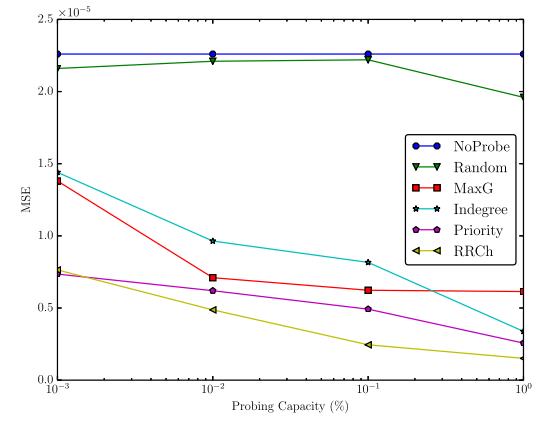

(a) Average MSE for all snapshots.
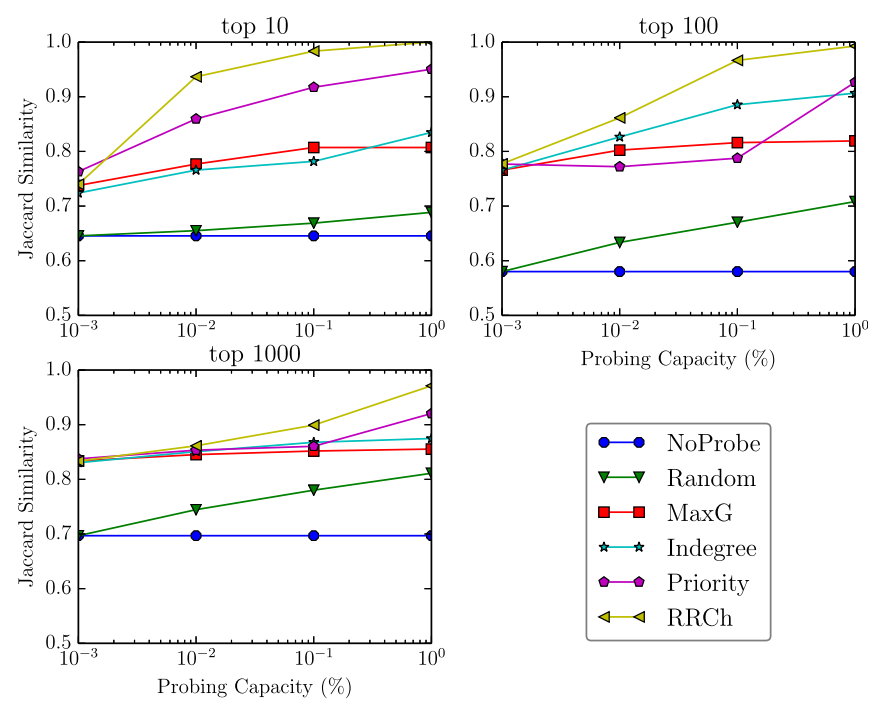

(b) Average Jaccard similarity for all snapshots.

Fig. 11. Comparison of the probing strategies.

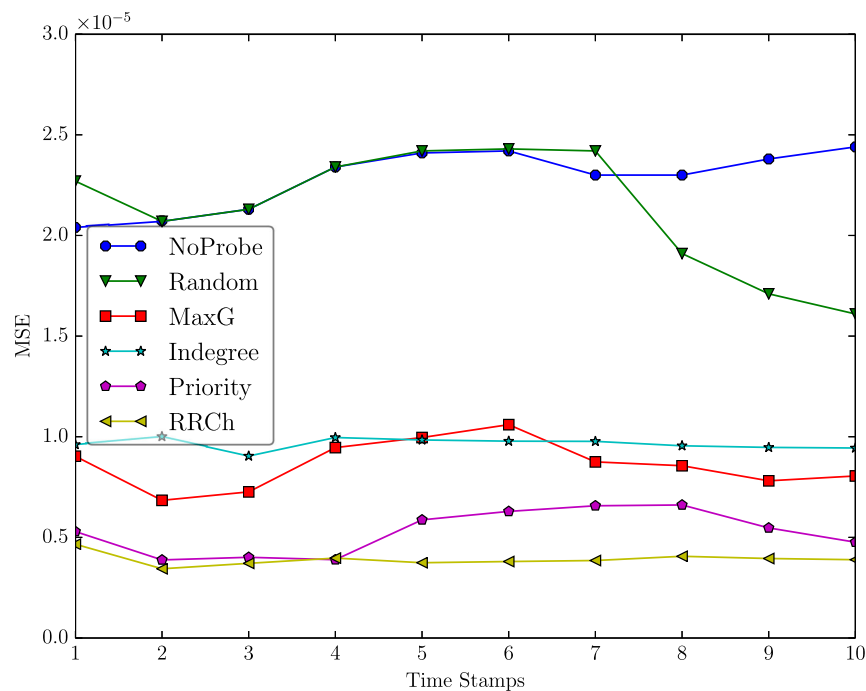

(a) Average MSE for all probing capacities.

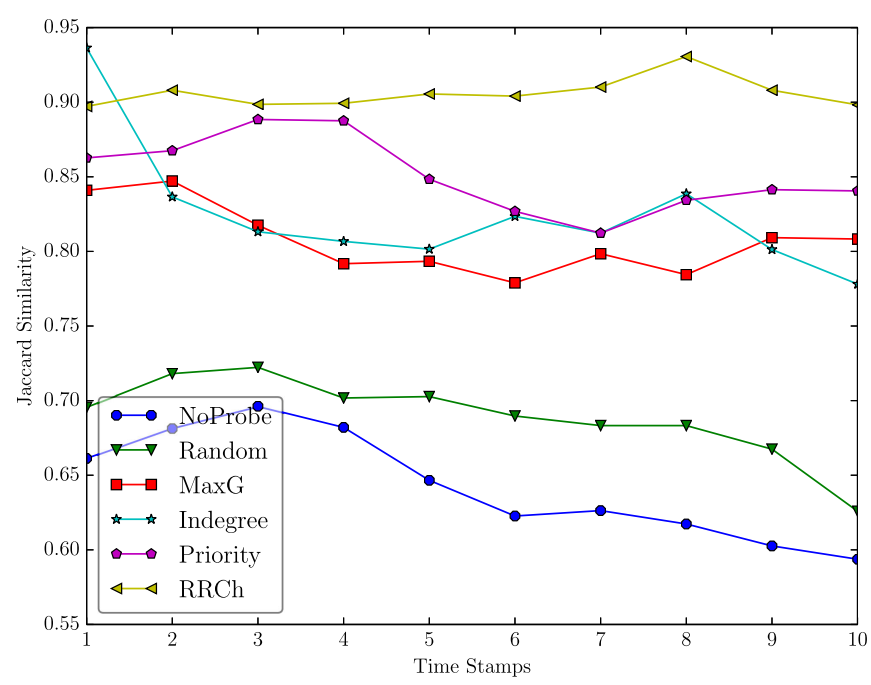

(b) Average Jaccard similarity for all probing capacities.

Fig. 12. Comparison of the probing strategies with respect to time.

is shown in Fig. 14. The histogram indicates the accuracy of the RA index used for network inference. The edges that were determined by the prediction algorithm as more likely to happen were found to be existent in the future network with a higher probability. However, when we analyzed the incorrectly predicted edges, we have observed that the algorithm predicts links between users who are unlikely to follow each other in real life. For example, the algorithms predict an edge between two pop stars since they have many common neighbors. However, they would not follow each other because they are main competitors. Furthermore, some of these users are not willing to follow anybody at all. This is the same issue studied in [12]. Link prediction algorithms typically do not consider these facts in social networks. In addition to indexes which they use to calculate similarities between users, they should also consider the tendency of the users to make new connections. Therefore, we apply a filtering process such that we only consider users who follow more than a threshold number of users in order to determine users who are likely to follow somebody. We add the predicted edges only to these selected users. As a result, we improve the RRCh method by 3 percent for 

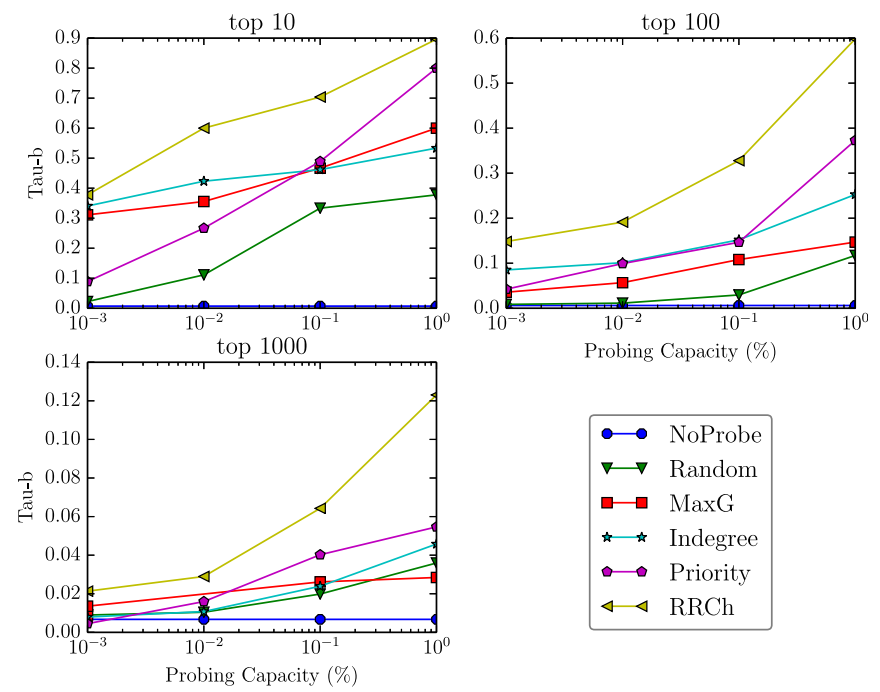

Fig. 13. Comparison of the probing strategies with respect to average Kendall Tau-b measure.

MSE and 2 percent for the set similarities on average. Since the improvements are not significant, we omit the plots of those results for brevity. Here, adaptation of more advanced (like mentioned in 4) prediction algorithms could potentially increase the accuracy of this technique. Moreover, the computational overhead of the link prediction task is not significant due to the pruning process applied for the experiments. The task takes less than a hour for one iteration. The time would significantly increase for the size of original networks.

\subsubsection{Evaluation of the Topic Influence Estimation}

We evaluated the influence of users with respect to four different topics: a) Politics, b) Sport, c) Health, and d) Cultural and Art Activities. This section provides a qualitative discussion about the accounts which were found to be influential by the proposed methods. Table 1 shows the accuracy of topic relevance of the top-10 users found by the system for the specific topics.

For the evaluation of the results, we performed a small survey containing 10 people chosen among graduate students

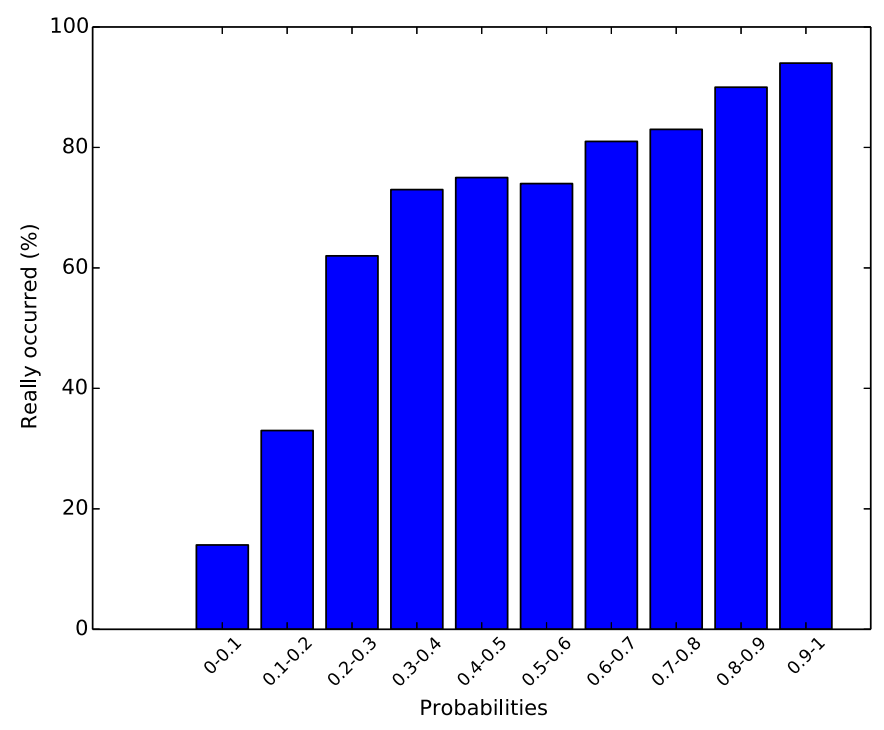

Fig. 14. Accuracy of the link prediction algorithm.
TABLE 1

Estimated Influential Accounts

\begin{tabular}{lcc}
\hline Topics & Topic Relevance & Some selected accounts \\
\hline Politics & 10 out of 10 & RT_Erdogan, kilicdarogluk, \\
O6melikgokcek \\
Sport & 8.5 out of 10 & $\begin{array}{c}\text { Fenerbahce, GalatasaraySK, } \\
\text { ntvspor }\end{array}$ \\
$\begin{array}{l}\text { Health } \\
\text { Cultural and } \\
\text { Art Activities }\end{array}$ & $\begin{array}{l}\text { 4 out of } 10 \\
\text { saglikbakanligi, YYD_tr, istabip } 10\end{array}$ & $\begin{array}{c}\text { CMYLMZ, AtlasTarihDergi, } \\
\text { Siirler_sokakta }\end{array}$ \\
\hline
\end{tabular}

who are closely interested in social media. We asked participants to evaluate the users with respect to their topic relevance and their influence on the topic. All participants were shown all influential account for all topics. In order to identify influence of a user, we asked participants to mark one of the following categories: $a$ ) very influential $(1), b$ ) influential (.5), c) not influential (0). Results are aggregated as average and rounded by .5 precision. We used the results of the survey to provide an evaluation of the selected users for the Turkish Twitter network, on a per-topic basis.

For the topic Politics, the results are very accurate for top10. We have observed that the dictionaries constructed for each topic has a big impact on the results. For example, we observe that the dictionary constructed for Politics topic contains many keywords that are related only with politics without any ambiguity. These keywords have increased the performance of the semantic analysis, which in turn increased the accuracy of the topic-based network influence analysis. Top-10 list contains the president of Turkish Republic (RT_Erdogan), the chairman of one of the opposition parties (kilicdarogluk), and the mayor of the capital city (06melikgokcek). It is fair to assume that these users, who give political messages in their tweets and who have lots of followers, should be in the top-10 influential list on Turkish Politics topic.

The influential accounts for the Sport topic were the biggest sport clubs of Turkey (Fenerbahce, GalatasaraySK) and one of the highest rating sport channel (ntvspor). Their tweets were mostly related to the sport competitions, news from clubs, etc. They have a lot of followers who actively pay attention to what they tweet. Thus, they achieve high RT and Fav statistics, which shows that they have a big impact on their followers. It is very reasonable that they are the top influential accounts on this topic.

As intuitively expected, the influential accounts for the Health topic are mostly doctor associations and governmental authorities. One of the accounts is Republic of Turkey Ministry of Health (saglikbakanligi), which mainly tweets about hospitals, doctors, and health regulations. Its follower numbers can be considered as relatively high and is followed by other influential accounts. Since its tweets have critical news potential, it has considerable number of RTs about the health topic. The other two are doctor associations (YYD_tr, istabip). They are followed by many doctors, which also have some potential impact on the Health topic. In this topic, accurate relevance ratio is relatively low because the constructed dictionary for this topic is not specific enough, causing errors in semantic analyses that propagates to the latter phase of influence estimation. 


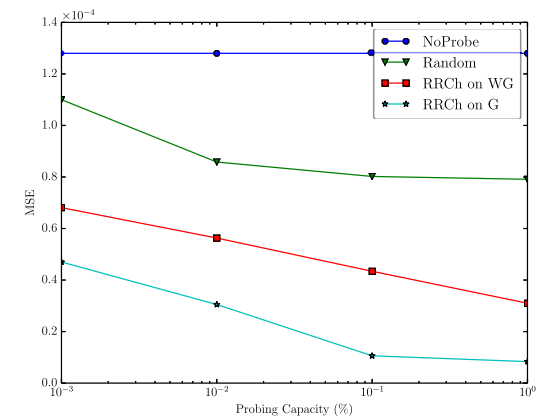

(a) MSE
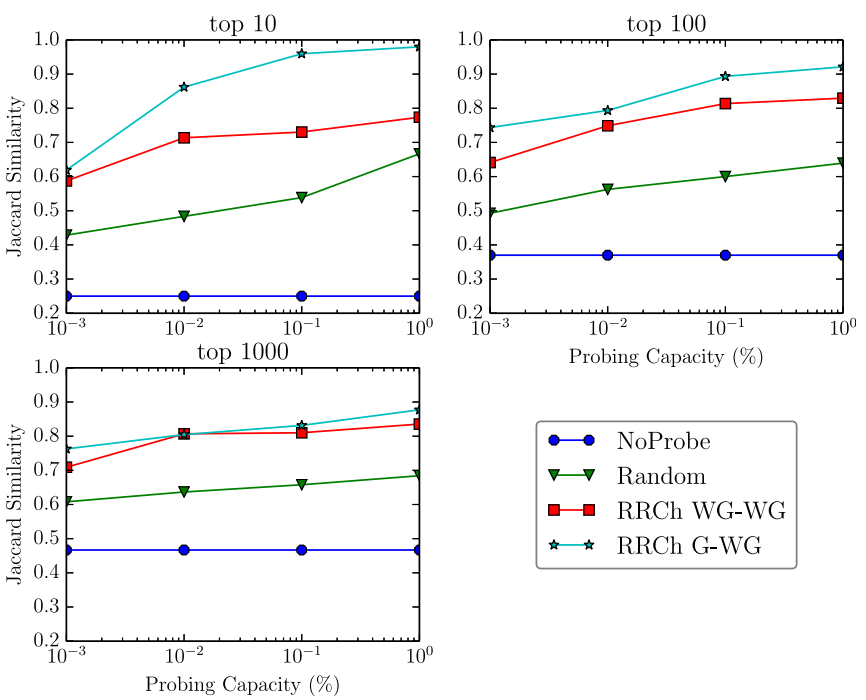

(b) Jaccard similarity

Fig. 15. Performance of change probing for dynamic tweet fetching.

The Cultural and Art Activities topic includes users which tweet about movies, art, books, history, etc. The top-10 influential users are perfectly matched with the keywords. CMYLMZ is a very famous Turkish comedian, actor and producer. He also has one of the highest follower numbers in the Turkish Twitter network. AtlasTarihDergi is a history magazine tweeting mainly about historical events and information which has considerable amount of followers and RTs. The third user (Siirler_sokakta) shares street poems and mottos, and it's posts receive many RTs and Favs.

\subsubsection{Evaluation of Dynamic Tweet Fetching}

We have used the same default parameter settings from the network fetching experiments to evaluate our proposed tweet fetching methods. For the simplicity, we only evaluate the case of topic Politics.

Fig. 15 shows the performance of the RRCh method for dynamic tweet fetching. For the MSE measure, global network based $G-W G$ method performs 78 percent better, and topic network based $W G-W G$ method performs 40 percent better than the baselines, on average, respectively. In Fig. 15b, we see that as the probing capacities increase, $G$-WG method achieves almost perfect similarity against the results obtained using the original network, for the top10 influential users. For the top-1000 influential users experiment, it reaches close to 0.9 similarity. Together with $W G-W G$ method, they quickly reach close to their top performance at around 1 percent capacity, except for the top-10
TABLE 2

Top-10 Topic Relevance Ratios for $G-W G$ and $W G-W G$ for Dynamic Tweet Fetching

\begin{tabular}{lcc}
\hline Topics & \multicolumn{2}{c}{ Topic Relevance } \\
\hline Politics & 10 out of 10 & 10 out of 10 \\
Sport & 8 out of 10 & 9 out of 10 \\
Health & 5 out of 10 & 4 out of 10 \\
Cultural and & 9 out of 10 & 9 out of 10 \\
Art Activities & $G$-WG method & $W G$-WG method \\
&
\end{tabular}

case. For the latter, $W G-W G$ method does not enjoy the quality increase that the $G-W G$ method enjoys with increasing capacities. When we look at the Jaccard similarity based results, $G-W G$ achieves 77 percent better and $W G-W G$ achieves 65 percent better results than the baselines. Overall, the results show us that using the globally maintained network is more advantageous.

Although $G-W G$ method outperforms $W G-W G$ method when we compare the top-10 results for the two methods, they are similar in terms of the topic relevance of their top influential users. Table 2 shows the topic relevance ratios for the two methods. Top-10 selected users are found to be related with the topics of interest and are popular accounts in the topic area.

\section{FUtURE DiRECTIONS}

In this section we discuss improvements and extensions to our work that are left as future research directions.

First, the simulation technique used in this study for evaluating the probing strategies does not take into account the following two aspects: i) a snapshot of the network collected as ground truth data does not represent an instantaneous snapshot and instead is the result of crawling, which takes nonnegligible amount of time, and ii) the simulation of probing strategies assumes that the network does not change as the probing happens, but in a real-world scenario the network can evolve during this time. A future direction for having more accurate simulation results is to consider the probing time explicitly as part of the simulation, while at the same time modeling the network change as a random process.

Second, this study focuses on effectively probing the network for capturing edge updates, which constitutes the majority of the change in the social network. Yet, node additions and deletions also take place in a dynamic network. Our proposed system handles node updates by periodically repeating the seed list construction process. We leave it as a future work to integrate node update into the edge probing process.

Third, for the topic-based network construction, we ignore the impact of individual tweets. We maintain a keyword corpora for user tweet sets and perform our topic analysis over these corpora. For approximating a user's influence on a particular topic, we scale her RT and FAV statistics with the relative relevance of her tweet set with the given topic. This is not as accurate as analyzing individual tweets. This is because a user may be tweeting mostly about one topic, yet receiving most of her RTs and FAVs for tweets posted about another topic. Integrating a topic 
classifier that works at the granularity of individual tweets is left as a future work. Luckily, such a classifier can be easily plugged into our framework. Similarly, topic classification techniques that are more advanced than the weighted keyword dictionaries we employed in this study can be integrated into our framework with ease.

Last, another interesting future research direction is utilizing a technique that can dynamically adjust $\theta$, which controls the balance between the last PageRank score and the change in recent PageRank scores in Eq. (7). Here, one can use an adaptive value at each iteration, tuned for each user based on some heuristic. An intelligent way of performing adaptive $\theta$ control could potentially improve the accuracy of the proposed techniques.

\section{Related Work}

Increases in the popularity of social networks and the availability of public data acquisition tools for them have put social networks on the spotlight of both academic and industrial research. Influential user estimation problem is studied by many researchers following a wide variety of different methodologies. Within this context, some studies introduce centrality measures in order to reflect influence of users. Wasserman [13] introduces several definitions, such as degree centrality, betweenness centrality, and closeness centrality. For viral marketing applications, [14] develops methods for computing network influence from collaborative filtering databases by using heuristics in a general descriptive probabilistic model of influence propagation. Kempe et al. [15] addresses a similar problem by studying the linear threshold and independent cascade models, and [16] presents a simple greedy algorithm for maximizing the spread of influence using a general model of social influence, termed the decreasing cascade model.

Recently, researchers have studied extracting textual information associated with social networks. Mei et al. [17] studies topic modeling in social networks and proposes a solution for text mining on the network structure. Tang et al. [18] introduces the topic-based social influence problem. Their proposed model takes the result of any predefined topic modeling of a social network and constructs a network representing topic-based influence propagation. Distributed learning algorithms are used for this purpose, which leverage the Map-Reduce concept. Thus, their methodology scales to large networks. Liu et al. [19] combines heterogeneous links and textual content for each user in order to mine topic-based influence. In another seminal work, [20] studies topic-specific influence by using PageRank.

Another recent study [21] uses a PageRank-like measure to find influential accounts on Twitter. They extend PageRank by using topic-specific probabilities in the random surfer model. Although their method is similar to ours, their influence measure utilizes the number of posts made on a specific topic. However, this is an indirect measure that cannot reliably capture influence. Therefore, we use topic distributions of user posts along with their sharing statistics (re-tweets and favorites in Twitter), which provides robust results, as it takes into account the real impact of posts. Hong and Davison [22] conducts an empirical study of different topic modeling strategies based on standard Latent Dirichlet Allocation (LDA) [23]. Lin et al. [24] proposes joint probabilistic models of influence and topics. Their methodology performs a topic sampling over textual contents and tracks the topic snapshots over time. Hong et al. [25] uses retweets in measuring popularity and proposes machine learning techniques to predict popularity of Twitter posts. Szabo and Huberman [26], Yang and Leskovec [27], Cheng et al. [28] propose solutions for predicting popularity of online content. Chen et al. [29] studies the topic-aware influence maximization problem. Within this context, in this work we introduce a new method that combines topic-based analyses of posts with their sharing popularity for the purpose of topic-based influential user estimation.

Dynamic graph analysis has also attracted a lot of attention recently. In order to maintain dynamic networks, [30], [31], [32], [33], [34] propose algorithms for determining web crawling schedules. Leskovec et al. [35] studies the microscopic evolution of social networks. Desikan and Pathak [36] studies incremental PageRank on evolving graphs. Researches have also investigated probing strategies for analyzing evolving social networks. Bahmani et al. [11] proposes influence proportional probing strategies for the computation of PageRank on evolving networks and [10] uses a probing strategy to capture observed image of the network by maximizing a performance gap function. Papagelis et al. [37], Valkanas et al. [38], Nazi et al. [39] study sampling over social networks. However, these studies only focus on current image of a network in their probing strategies. In contrast, we propose a method which also considers evolution of the probing metrics, so that the network could be probed more effectively.

In the context of network inference, [40] proposes representations for structural uncertainty and use directed graphical models and probabilistic relational models for link structure learning. However, their methodologies are not scalable. Ghahramani [41], Song et al. [42], Koskinen and Snijders [43] use time evolving graph models for social network estimation. They apply time-varying dynamic Bayesian networks for modeling evolving network structures. Bonneau et al. [44] shows that third-parties can reach a user's information by searching a few friends. Rodriguez et al. [45] develops a scalable algorithm to infer influence and diffusion network based on an assumption that all users in the network influence their neighbors with equal probability. Myers and Leskovec [46] removes this assumption and addresses the more general problem by formulating a maximum likelihood problem and guarantee the optimality of the solution. Yang and Leskovec [47] proposes a linear model to predict how diffusion unfolds over time and [48] proposes the notion of diffusion centrality. Yang and Leskovec [49], Rodriguez et al. [50] studies a different problem related to network inference. Different from these works, we use friendship weighting method in order to infer link structures, similar to [51], [52], [53]. However, we use friendship weights only to infer edges between users. Du et al. [54] proposes a kernel based method and [55] uses a continuous time model for inference. Moreover, one can also use more informative features such as content-based influential effects. Wang et al. [56] studies diffusion of tweets throughout the Twitter network. This kind of technique could also be used in order to estimate impact of posts. 


\section{CONCLUSION}

The rate restrictions enforced by social network service providers have a negative impact on the third-party evolving network analysis tasks. Therefore, we proposed probing algorithms to dynamically fetch network topology and text data from social networks under limited probing capacities. Our proposed solutions use the past influence trends of the users, as well as their current influences, in order to determine the best users to probe, with the aim of maximizing the influence estimation accuracy. In particular, we observed that highly influential users and users with strong influence trends affect the overall influence estimations the most. We have leveraged these two metrics across our probing algorithms. Experimental results have shown that considering past trends in the probing strategy increases the overall accuracy of influence prediction. Furthermore, we improved our probing strategies by inferring possible relations between users via link prediction algorithms. We also developed techniques for estimating topic-based user influence in dynamic social networks. For computing topicbased influence, we proposed methods that consider both the place of the user in the network topology, as well as the topic analysis performed on the user posts and the sharing statistics of these posts. Our experimental results performed on Twitter network data has shown improved accuracy compared to state-of-the-art methods from the literature.

\section{ACKNOWLEDGMENTS}

Special thanks to Mr. Mehmet Güvercin for his contributions to creation of the word dictionaries and topic relevance scoring. This work is supported in part by Turkish Academy of Sciences and Türk Telekom.

\section{REFERENCES}

[1] Twitter, 2006. [Online]. Available: https:/ / twitter.com/

[2] Twitter API rate limits, 2015. [Online]. Available: https://dev. twitter.com/rest/public/rate-limiting

[3] L. Page, S. Brin, R. Motwani, and T. Winograd, "The PageRank citation ranking: Bringing order to the Web," Stanford InfoLab, 1999-66, Stanford, CA, USA, 1999

[4] W. Xing and A. Ghorbani, "Weighted PageRank algorithm," in Proc. 2nd Annu. Conf. Commun. Netw. Services Res., 2004, pp. 305-314.

[5] M. Naaman, J. Boase, and C.-H. Lai, "Is it really about me?: Message content in social awareness streams," in Proc. ACM Conf. Comput. Supported Cooperative Work, 2010, pp. 189-192. [Online]. Available: http://doi.acm.org/10.1145/1718918.1718953

[6] P. Analytics, "Twitter study-august2009," San Antonio, TX: Pear Analytics, 2009. [Online]. Available: www.pearanalytics.com/ blog/wp-content/uploads/2010/05/Twitter-Study-August-2009. pdf, 2009 .

[7] M. Hibon and S. Makridakis, "ARMA models and the box-jenkins methodology," J. Forecasting, vol. 16, pp. 147-163, 1997.

[8] L. Lü and T. Zhou, "Link prediction in complex networks: A survey," Physica A: Statist. Mech. Appl., vol. 390, no. 6, pp. 1150-1170, 2011.

[9] L. Backstrom and J. Leskovec, "Supervised random walks: Predicting and recommending links in social networks," in Proc. 4th ACM Int. Conf. Web Search Data Mining, 2011, pp. 635-644.

[10] H. Zhuang, Y. Sun, J. Tang, J. Zhang, and X. Sun, "Influence maximization in dynamic social networks," in Proc. IEEE 13th Int. Conf. Data Mining, Dec. 2013, pp. 1313-1318.

[11] B. Bahmani, R. Kumar, M. Mahdian, and E. Upfal, "PageRank on an evolving graph," in Proc. 18th ACM SIGKDD Int. Conf. Knowl. Discovery Data Mining, 2012, pp. 24-32.

[12] H. Li, S. S. Bhowmick, and A. Sun, "CASINO: Towards conformity-aware social influence analysis in online social networks," in Proc. 20th ACM Int. Conf. Inf. Knowl. Manage., 2011, pp. 1007-1012.
[13] S. Wasserman, Social Network Analysis: Methods and Applications, vol. 8. Cambridge, U.K.: Cambridge Univ. Press, 1994.

[14] P. Domingos and M. Richardson, "Mining the network value of customers," in Proc. 7th ACM SIGKDD Int. Conf. Knowl. Discovery Data Mining, 2001, pp. 57-66.

[15] D. Kempe, J. Kleinberg, and E. Tardos, "Maximizing the spread of influence through a social network," in Proc. 9th ACM SIGKDD Int. Conf. Knowl. Discovery Data Mining, 2003, pp. 137-146.

[16] D. Kempe, J. Kleinberg, and É. Tardos, "Influential nodes in a diffusion model for social networks," in Automata, Languages and Programming. Berlin, Germany: Springer, 2005, pp. 1127-1138.

[17] Q. Mei, D. Cai, D. Zhang, and C. Zhai, "Topic modeling with network regularization," in Proc. 17th Int. Conf. World Wide Web, 2008, pp. 101-110.

[18] J. Tang, J. Sun, C. Wang, and Z. Yang, "Social influence analysis in large-scale networks," in Proc. 15th ACM SIGKDD Int. Conf. Knowl. Discovery Data Mining, 2009, pp. 807-816.

[19] L. Liu, J. Tang, J. Han, M. Jiang, and S. Yang, "Mining topic-level influence in heterogeneous networks," in Proc. 19th ACM Int. Conf. Inf. Knowl. Manage., 2010, pp. 199-208.

[20] T. H. Haveliwala, "Topic-sensitive PageRank," in Proc. 11th Int. Conf. World Wide Web, 2002, pp. 517-526.

[21] J. Weng, E.-P. Lim, J. Jiang, and Q. He, "TwitterRank: Finding topic-sensitive influential twitterers," in Proc. 3rd ACM Int. Conf. Web Search Data Mining, 2010, pp. 261-270.

[22] L. Hong and B. D. Davison, "Empirical study of topic modeling in twitter," in Proc. 1st Workshop Social Media Anal., 2010, pp. 80-88. [Online]. Available: http://doi.acm.org/10.1145/1964858.1964870

[23] D. M. Blei, A. Y. Ng, and M. I. Jordan, "Latent Dirichlet allocation," J. Mach. Learn. Res., vol. 3, pp. 993-1022, 2003.

[24] C. X. Lin, Q. Mei, J. Han, Y. Jiang, and M. Danilevsky, "The joint inference of topic diffusion and evolution in social communities," in Proc. IEEE 11th Int. Conf. Data Mining, 2011, pp. 378-387.

[25] L. Hong, O. Dan, and B. D. Davison, "Predicting popular messages in twitter," in Proc. 20th Int. Conf. Companion World Wide Web, 2011, pp. 57-58.

[26] G. Szabo and B. A. Huberman, "Predicting the popularity of online content," Commun. ACM, vol. 53, no. 8, pp. 80-88, Aug. 2010. [Online]. Available: http://doi.acm.org/10.1145/1787234.1787254

[27] J. Yang and J. Leskovec, "Patterns of temporal variation in online media," in Proc. 4th ACM Int. Conf. Web Search Data Mining, 2011, pp. 177-186. [Online]. Available: http://doi.acm.org/10.1145/ 1935826.1935863

[28] J. Cheng, L. Adamic, P. A. Dow, J. M. Kleinberg, and J. Leskovec, "Can cascades be predicted?" in Proc. 23rd Int. Conf. World Wide Web, 2014, pp. 925-936.

[29] S. Chen, J. Fan, G. Li, J. Feng, K.-L. Tan, and J. Tang, “Online topicaware influence maximization," Proc. VLDB Endowment, vol. 8, no. 6, pp. 666-677, 2015.

[30] J. L. Wolf, M. S. Squillante, P. Yu, J. Sethuraman, and L. Ozsen, "Optimal crawling strategies for Web search engines," in Proc. 11th Int. Conf. World Wide Web, 2002, pp. 136-147.

[31] J. Cho and H. Garcia-Molina, "Effective page refresh policies for Web crawlers," ACM Trans. Database Syst., vol. 28, no. 4, pp. 390426, Dec. 2003. [Online]. Available: http://doi.acm.org/10.1145/ 958942.958945

[32] J. Cho and H. Garcia-Molina, "Estimating frequency of change," ACM Trans. Internet Technol., vol. 3, no. 3, pp. 256-290, 2003.

[33] S. Pandey and C. Olston, "User-centric Web crawling," in Proc. 14th Int. Conf. World Wide Web, 2005, pp. 401-411. [Online]. Available: http://doi.acm.org/10.1145/1060745.1060805

[34] C. Olston and S. Pandey, "Recrawl scheduling based on information longevity," in Proc. 17th Int. Conf. World Wide Web, 2008, pp. 437-446. [Online]. Available: http://doi.acm.org/10.1145/ 1367497.1367557

[35] J. Leskovec, L. Backstrom, R. Kumar, and A. Tomkins, "Microscopic evolution of social networks," in Proc. 14th ACM SIGKDD Int. Conf. Knowledge Discovery Data Mining, 2008, pp. 462-470.

[36] P. Desikan and N. Pathak, "Incremental PageRank computation on evolving graphs," in Proc. Int. World Wide Web Conf., 2005, pp. $10-14$.

[37] M. Papagelis, G. Das, and N. Koudas, "Sampling online social networks," IEEE Trans. Knowl. Data Eng., vol. 25, no. 3, pp. 662676, Mar. 2013.

[38] G. Valkanas, I. Katakis, D. Gunopulos, and A. Stefanidis, “Mining twitter data with resource constraints," in Proc. IEEE/WIC/ACM Int. Joint Conf. Web Intell. Intell. Agent Technol., 2014, pp. 157-164. [Online]. Available: http://dx.doi.org/10.1109/WI-IAT.2014.29 
[39] A. Nazi, Z. Zhou, S. Thirumuruganathan, N. Zhang, and G. Das, "Walk, not wait: Faster sampling over online social networks," in Proc. VLDB Endowment, vol. 8, no. 6, pp. 678-689, 2015.

[40] L. Getoor, N. Friedman, D. Koller, and B. Taskar, "Learning probabilistic models of link structure," J. Mach. Learn. Res., vol. 3, pp. 679-707, 2003.

[41] Z. Ghahramani, "Learning dynamic Bayesian networks," in Adaptive Processing of Sequences and Data Structures. Berlin, Germany: Springer, 1998, pp. 168-197.

[42] L. Song, M. Kolar, and E. P. Xing, "Time-varying dynamic Bayesian networks," in Proc. Advances Neural Inf. Process. Syst., 2009, pp. 1732-1740.

[43] J. H. Koskinen and T. A. Snijders, "Bayesian inference for dynamic social network data," J. Statist. Planning Inference, vol. 137, no. 12, pp. 3930-3938, 2007.

[44] J. Bonneau, J. Anderson, R. Anderson, and F. Stajano, "Eight friends are enough: Social graph approximation via public listings," in Proc. 2nd ACM EuroSys Workshop Social Netw. Syst., 2009, pp. 13-18.

[45] M. Gomez Rodriguez, J. Leskovec, and A. Krause, "Inferring networks of diffusion and influence," in Proc. 16th ACM SIGKDD Int. Conf. Knowl. Discovery Data Mining, 2010, pp. 1019-1028.

[46] S. Myers and J. Leskovec, "On the convexity of latent social network inference," in Proc. Advances Neural Inf. Process. Syst., 2010, pp. 1741-1749.

[47] J. Yang and J. Leskovec, "Modeling information diffusion in implicit networks," in Proc. IEEE Int. Conf. Data Mining, 2010, pp. 599-608. [Online]. Available: http://dx.doi.org/10.1109/ ICDM.2010.22

[48] C. Kang, C. Molinaro, S. Kraus, Y. Shavitt, and V. Subrahmanian, "Diffusion centrality in social networks," in Proc. Int. Conf. Advances Social Netw. Anal. Mining, 2012, pp. 558-564.

[49] J. Yang and J. Leskovec, "Modeling information diffusion in implicit networks," in Proc. IEEE 10th Int. Conf. Data Mining, 2010, pp. 599-608.

[50] M. G. Rodriguez, D. Balduzzi, and B. Schölkopf, “Uncovering the temporal dynamics of diffusion networks," arXiv:1105.0697, 2011.

[51] B. Taskar, M.-F. Wong, P. Abbeel, and D. Koller, "Link prediction in relational data," in Proc. Advances Neural Inf. Process. Syst., 2003, pp. 659-666.

[52] J.-P. Vert and Y. Yamanishi, "Supervised graph inference," in Proc. Advances Neural Inf. Process. Syst., 2004, pp. 1433-1440.

[53] D. Liben-Nowell andJ. Kleinberg, "The link-prediction problem for social networks," J. Amer. Soc. Inf. Sci. Technol., vol. 58, no. 7, pp. 1019-1031, 2007.

[54] N. Du, L. Song, M. Yuan, and A. J. Smola, "Learning networks of heterogeneous influence," in Proc. Advances Neural Inf. Process. Syst., 2012, pp. 2780-2788.

[55] N. Du, L. Song, H. Woo, and H. Zha, "Uncover topic-sensitive information diffusion networks," in Proc. 16th Int. Conf. Artif. Intell. Statist., 2013, pp. 229-237.

[56] B. Wang, et al., "Whom to mention: Expand the diffusion of tweets by@ recommendation on micro-blogging systems," in Proc. 22nd Int. Conf. World Wide Web, 2013, pp. 1331-1340.

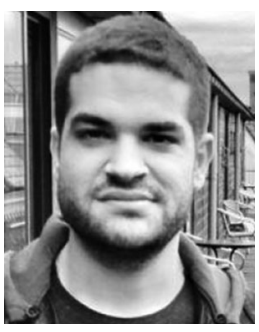

Kaan Bingöl is working toward the MSc degree in the Department of Computer Engineering, Bilkent University, Turkey. His research interests include large dynamic graphs and big data technologies.

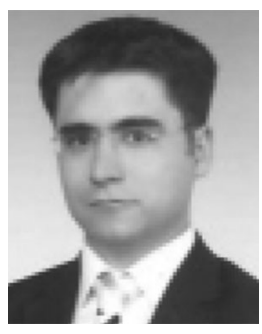

Bahaeddin Eravcı is working toward the $\mathrm{PhD}$ degree in computer science at Bilkent University. His research interests include time series data mining and data management.

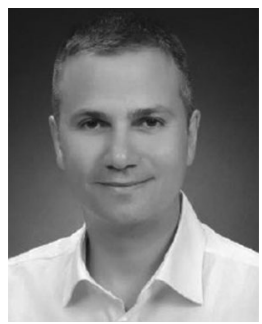

Çağrı Özgenç Etemoğlu received the $\mathrm{PhD}$ degree from the University of California, Santa Barbara. He is the manager with Big Data Software Development Group, Türk Telekom. His research interest includes big data systems.

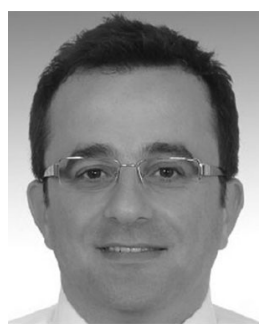

Hakan Ferhatosmanoglu received the $\mathrm{PhD}$ degree in computer science from the University of California, Santa Barbara, in 2001. He is a professor with Bilkent University, Turkey. He was with Ohio State University before joining Bilkent. His research interests include scalable management and analysis of streaming and multi-dimensional data. He received the Career awards from the US Department of Energy, US National Science Foundation, and Turkish Academy of Sciences.

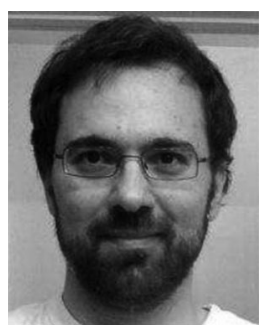

Bugra Gedik received the PhD degree in computer science from Georgia Tech. He is an associate professor in the Department of Computer Engineering, Bilkent University, Turkey. His research interests include distributed data-intensive systems, with a particular focus on stream computing, and big data technologies. 